\title{
A Geometric Approach to the Optimal Control of Nonholonomic Mechanical Systems
}

\author{
Anthony Bloch, Leonardo Colombo, Rohit Gupta, and David Martín de Diego
}

\begin{abstract}
In this paper, we describe a constrained Lagrangian and Hamiltonian formalism for the optimal control of nonholonomic mechanical systems. In particular, we aim to minimize a cost functional, given initial and final conditions where the controlled dynamics are given by a nonholonomic mechanical system. In our paper, the controlled equations are derived using a basis of vector fields adapted to the nonholonomic distribution and the Riemannian metric determined by the kinetic energy. Given a cost function, the optimal control problem is understood as a constrained problem or equivalently, under some mild regularity conditions, as a Hamiltonian problem on the cotangent bundle of the nonholonomic distribution. A suitable Lagrangian submanifold is also shown to lead to the correct dynamics. Application of the theory is demonstrated through several examples including optimal control of the Chaplygin sleigh, a continuously variable transmission, and a problem of motion planning for obstacle avoidance.
\end{abstract}

\section{Introduction}

Although nonholonomic systems have been studied since the dawn of analytical mechanics, there has been some confusion over the correct formulation of the equations of motion (see, e.g., [4, 10] and [29] for some of the history). It is only recently that their geometric formulation has been fully understood. In addition, there has been recent interest in the analysis of control problems for such systems.

\footnotetext{
A. Bloch $(\bowtie) \cdot$ L. Colombo

Department of Mathematics, University of Michigan, Ann Arbor, MI 48109, USA

e-mail: abloch@umich.edu; ljcolomb@umich.edu

R. Gupta

Department of Aerospace Engineering, University of Michigan, Ann Arbor, MI 48109, USA

e-mail: rohitgpt@umich.edu

D.M. de Diego

Instituto de Ciencias Matemáticas (CSIC-UAM-UC3M-UCM), C. Nicolás Cabrera 15, 28049

Madrid, Spain

e-mail: david.martin@icmat.es
} 
Nonholonomic control systems exhibit distinctive features. In particular, many naturally underactuated systems are controllable, the controllability arising from the nonintegrability of the constraints.

Nonholonomic optimal control problems arise in many engineering applications, for instance systems with wheels, such as cars and bicycles, systems with blades or skates, etc. There are thus multiple applications in the context of wheeled motion, space or mobile robotics and robotic manipulation. In this paper, we will introduce some new geometric techniques in nonholonomic mechanics to study the case of control effort minimizing optimal control problems.

The application of modern tools from differential geometry in the fields of mechanics, control theory, field theory and numerical integration has led to significant progress in these research areas. For instance, the study of the geometrical formulation of the nonholonomic equations of motion has led to better understanding of different engineering problems such as locomotion generation, controllability, motion planning, and trajectory tracking (see, e.g., [4-8, 12, 13, 22, 25, 30$32,37,39$ ] and references therein). Geometric techniques can also be used to study optimal control problems (see, e.g., [8, 15, 16, 20, 21, 42, 43]).

Combining these ideas in this paper, we study the underlying geometry of optimal control problems for mechanical systems subject to nonholonomic constraints and we apply it to several interesting examples.

Classical nonholonomic constraints which are linear in the velocities can be geometrically encoded by a constant rank distribution $\mathcal{D}$. As we will see, the distribution $\mathcal{D}$ will play the role of the velocity phase space. Given a Lagrangian $L=K-V: T Q \rightarrow \mathbb{R}$, where $K$ and $V$ are the kinetic and potential energy, respectively, and a distribution $\mathcal{D}$ where the motion of the system is restricted, the dynamics of the nonholonomic system is completely determined using the Lagrange-d'Alembert principle [4]. In this paper, we will formulate a description in terms of a Levi Civita connection defined on the space of vector fields taking values on $\mathcal{D}$. This connection is obtained by projecting the standard Lie bracket using the Riemannian metric associated with the kinetic energy $K$ (see [3]) and the typical characterization of the Levi-Civita connection (see also [9]). By adding controls in this setting we can study the associated optimal control problem. Moreover, we can see that the dynamics of the optimal control problem is completely described by a Lagrangian submanifold of an appropriate cotangent bundle and, under some regularity conditions, the equations of motion are derived as classical Hamilton's equations on the cotangent bundle of the distribution, $T^{*} \mathcal{D}$. Although our approach is quite intrinsic, we also give a local description since this is important for working out examples. For this, it is necessary to choose an adapted basis of vector fields for the distribution. From this point of view, we combine the techniques used previously by the authors of the paper (see $[3,11,35]$ ). An additional advantage of our method is that symmetries may be naturally analyzed in this setting. Concretely, the main results of our paper can be summarized as follows:

- Geometric derivation of the equations of motion of nonholonomic optimal control problems as a constrained problem on the tangent space of the constraint distribution $\mathcal{D}$. 
- Construction of a Lagrangian submanifold representing the dynamics of the optimal control problem and the corresponding Hamiltonian representation when the system is regular.

- Definition of a Legendre transformation establishing the relationship and correspondence between the Lagrangian and Hamiltonian dynamics.

- The application of the theory is demonstrated through several examples including optimal control of the Chaplygin sleigh, a continuously variable transmission and a problem of motion planning for obstacle avoidance.

\section{Nonholonomic Mechanical Systems}

Constraints on mechanical systems are typically divided into two types: holonomic and nonholonomic, depending on whether the constraint can be derived from a constraint in the configuration space or not. Therefore, the dimension of the space of configurations is reduced by holonomic constraints but not by nonholonomic constraints. Thus, holonomic constraints allow a reduction in the number of coordinates of the configuration space needed to formulate a given problem (see [38]).

We will restrict ourselves to the case of nonholonomic constraints. Additionally, assume that the constraints are given by a nonintegrable distribution $\mathcal{D}$ on the configuration space $Q$. If we choose local coordinates $\left(q^{i}\right), 1 \leq i \leq n=\operatorname{dim} Q$, the linear constraints on the velocities are locally given by equations of the form

$$
\phi^{a}\left(q^{i}, \dot{q}^{i}\right)=\mu_{i}^{a}(q) \dot{q}^{i}=0, \quad 1 \leq a \leq m \leq n,
$$

depending, in general, on local coordinates and their velocities. From an intrinsic point of view, the linear constraints are defined by a distribution $\mathcal{D}$ on $Q$ of constant rank $n-m$ such that the annihilator of $\mathcal{D}$ is locally given by

$$
\operatorname{Ann}(\mathcal{D})=\operatorname{span}\left\{\mu^{a}=\mu_{i}^{a} d q^{i} ; 1 \leq a \leq m\right\}
$$

where the 1-forms $\mu^{a}$ are independent.

In addition to these constraints, we need to specify the dynamical evolution of the system, usually by fixing a Lagrangian function $L: T Q \rightarrow \mathbb{R}$. In mechanics, the central concepts permitting the extension of mechanics from the Newtonian point of view to the Lagrangian one are the notions of virtual displacements and virtual work; these concepts were originally formulated and developed in mechanics for their use in statics. In nonholonomic dynamics, the dynamics is given by the Lagranged'Alembert principle. This principle allows us to determine the set of possible values of the constraint forces from the set $\mathcal{D}$ of admissible kinematic states alone. The resulting equations of motion are

$$
\left[\frac{d}{d t}\left(\frac{\partial L}{\partial \dot{q}^{i}}\right)-\frac{\partial L}{\partial q^{i}}\right] \delta q^{i}=0,
$$


where $\delta q^{i}$ denotes the virtual displacement satisfying

$$
\mu_{i}^{a} \delta q^{i}=0
$$

(for the sake of simplicity, we will assume that the system is not subject to nonconservative forces). This must be supplemented by the constraint equations (1). By using the Lagrange multiplier rule, we obtain

$$
\frac{d}{d t}\left(\frac{\partial L}{\partial \dot{q}^{i}}\right)-\frac{\partial L}{\partial q^{i}}=\lambda_{a} \mu_{i}^{a} .
$$

The term on the right hand side represents the constraint force or reaction force induced by the constraints. The functions $\lambda_{a}$ are Lagrange multipliers which, after being computed using the constraint equations, allow us to obtain a set of second order differential equations.

Now, we restrict ourselves to the case of nonholonomic mechanical systems where the Lagrangian is of mechanical type, given as follows

$$
L\left(v_{q}\right)=\frac{1}{2} \mathcal{G}\left(v_{q}, v_{q}\right)-V(q),
$$

where $v_{q} \in T_{q} Q$. Here $\mathcal{G}$ denotes a Riemannian metric on the configuration space $Q$ representing the kinetic energy of the system and $V: Q \rightarrow \mathbb{R}$ is the potential energy. Locally, the metric is determined by the matrix $M=\left(\mathcal{G}_{i j}\right)_{1 \leq i, j \leq n}$ where $\mathcal{G}_{i j}=\mathcal{G}\left(\frac{\partial}{\partial q^{i}}, \frac{\partial}{\partial q^{j}}\right)$.

Denote by $\tau_{\mathcal{D}}: \mathcal{D} \rightarrow Q$ the canonical projection of $\mathcal{D}$ to $Q$ and $\Gamma\left(\tau_{\mathcal{D}}\right)$ the set of sections of the bundle $\tau_{D}$, which is just the set of vector fields $\mathfrak{X}(Q)$ taking values on $\mathcal{D}$ in this context. If $X, Y \in \mathfrak{X}(Q)$, then $[X, Y]$ denotes the standard Lie bracket of the vector fields.

Definition 1 A nonholonomic mechanical system on a manifold $Q$ is given by the triple $(\mathcal{G}, V, \mathcal{D})$, where $\mathcal{G}$ is a Riemannian metric on $Q$, specifying the kinetic energy of the system, $V: Q \rightarrow \mathbb{R}$ is a smooth function representing the potential energy and $\mathcal{D}$ is a non-integrable distribution on $Q$ representing the nonholonomic constraints.

Remark 1 Given $X, Y \in \Gamma\left(\tau_{\mathcal{D}}\right)$ that is, $X(x) \in \mathcal{D}_{x}$ and $Y(x) \in \mathcal{D}_{x}$, for all $x \in Q$, then it may happen that $[X, Y] \notin \Gamma\left(\tau_{\mathcal{D}}\right)$ since $\mathcal{D}$ is nonintegrable.

We want to obtain a bracket defined for sections of $\tau_{\mathcal{D}}$. Using the Riemannian metric $\mathcal{G}$ we can construct two complementary orthogonal projection maps

$$
\mathcal{P}: T Q \rightarrow \mathcal{D} \text { and } Q: T Q \rightarrow \mathcal{D}^{\perp}
$$

with respect to the tangent bundle orthogonal decomposition $\mathcal{D} \oplus \mathcal{D}^{\perp}=T Q$. 
Therefore, given $X, Y \in \Gamma\left(\tau_{\mathcal{D}}\right)$ we define the nonholonomic bracket $\llbracket \cdot, \cdot \rrbracket$ : $\Gamma\left(\tau_{\mathcal{D}}\right) \times \Gamma\left(\tau_{\mathcal{D}}\right) \rightarrow \Gamma\left(\tau_{\mathcal{D}}\right)$ as

$$
\llbracket X, Y \rrbracket=\mathcal{P}[X, Y]
$$

where $X, Y \in \Gamma\left(\tau_{\mathcal{D}}\right)$ (see $[2,3,18]$ ). This Lie bracket satisfies all the usual properties of a Lie bracket except for the Jacobi identity.

Remark 2 From a more differential geometric point of view, $\mathcal{D}$ with this modified bracket for sections of the vector bundle $\tau_{\mathcal{D}}$, inherits a skew-symmetric Lie algebroid structure (see [17] and [3]), where now the bracket for sections of the vector bundle $\tau_{\mathcal{D}}$ does not satisfy in general the Jacobi identity, as an expression of the nonintegrability of the distribution $\mathcal{D}$.

Definition 2 Considering the restriction of the Riemannian metric $\mathcal{G}$ to the distribution $\mathcal{D}, \mathcal{G}^{\mathcal{D}}: \mathcal{D} \times_{Q} \mathcal{D} \rightarrow \mathbb{R}$ we can define the Levi-Civita connection

$$
\nabla^{\mathcal{G}^{\mathcal{D}}}: \Gamma\left(\tau_{\mathcal{D}}\right) \times \Gamma\left(\tau_{\mathcal{D}}\right) \rightarrow \Gamma\left(\tau_{\mathcal{D}}\right)
$$

determined by the following two properties:

1. $\llbracket X, Y \rrbracket=\nabla_{X}^{\mathcal{G}^{\mathcal{D}}} Y-\nabla_{Y}^{\mathcal{G}^{\mathcal{D}}} X \quad$ (Symmetry).

2. $X\left(\mathcal{G}^{\mathcal{D}}(Y, Z)\right)=\mathcal{G}^{\mathcal{D}}\left(\nabla_{X}^{\mathcal{G}^{\mathcal{D}}} Y, Z\right)+\mathcal{G}^{\mathcal{D}}\left(Y, \nabla_{X}^{\mathcal{G}^{\mathcal{D}}} Z\right)$ (Metricity).

Let $\left(q^{i}\right)$ be local coordinates on $Q$ and $\left\{e_{A}\right\}$ vector fields on $\Gamma\left(\tau_{D}\right)$ (that is, $e_{A}(x) \in$ $\mathcal{D}_{x}$ ), such that $\mathcal{D}_{x}=\operatorname{span}\left\{e_{A}(x)\right\}$, with $x \in U \subset Q$. The Christoffel symbols $\Gamma_{B C}^{A}$ of the connection $\nabla^{\mathcal{D}}$ are given by

$$
\nabla_{e_{B}}^{\mathcal{G}^{\mathcal{D}}} e_{C}=\Gamma_{B C}^{A}(q) e_{A}
$$

Definition 3 A curve $\gamma: I \subset \mathbb{R} \rightarrow \mathcal{D}$ is admissible if there exists a curve $\sigma: I \subset$ $\mathbb{R} \rightarrow Q$ projecting $\gamma$ to $Q$ such that $\gamma(t)=\dot{\sigma}(t)$.

Given local coordinates on $Q,\left(q^{i}\right) i=1, \ldots, n$, and $\left\{e_{A}\right\}$ a basis of sections of $\Gamma\left(\tau_{\mathcal{D}}\right)$, such that $e_{A}=\rho_{A}^{i}(q) \frac{\partial}{\partial q^{i}}$, we introduce induced coordinates $\left(q^{i}, y^{A}\right)$ on $\mathcal{D}$ where, if $e \in \mathcal{D}_{x}$ then $e=y^{A} e_{A}(x)$. Therefore, $\gamma(t)=\left(q^{i}(t), y^{A}(t)\right)$ is admissible if

$$
\dot{q}^{i}(t)=\rho_{A}^{i}(q(t)) y^{A}(t)
$$

Now, consider the restricted Lagrangian, $\ell: \mathcal{D} \rightarrow \mathbb{R}$,

$$
\ell(v)=\frac{1}{2} \mathcal{G}^{\mathcal{D}}(v, v)-V\left(\tau_{D}(v)\right)
$$

where $v \in \mathcal{D}$. 
Definition 4 (see [3]) A solution of the nonholonomic system is an admissible curve $\gamma: I \rightarrow \mathcal{D}$ such that

$$
\nabla_{\gamma(t)}^{\mathcal{G}^{\mathcal{D}}} \gamma(t)+\operatorname{grad}_{\mathcal{G} \mathcal{D}} V\left(\tau_{\mathcal{D}}(\gamma(t))\right)=0
$$

Here the section $\operatorname{grad}_{\mathcal{G} \mathcal{D}} V \in \Gamma\left(\tau_{\mathcal{D}}\right)$ is characterized by

$$
\mathcal{G}^{\mathcal{D}}\left(\operatorname{grad}_{\mathcal{G}^{\mathcal{D}}} V, X\right)=X(V)
$$

for every $X \in \Gamma\left(\tau_{\mathcal{D}}\right)$.

These equations are equivalent to the nonholonomic equations. Locally, these are given by

$$
\begin{aligned}
\dot{q}^{i} & =\rho_{A}^{i}(q) y^{A}, \\
\dot{y}^{C} & =-\Gamma_{A B}^{C} y^{A} y^{B}-\left(\mathcal{G}^{\mathcal{D}}\right)^{C B} \rho_{B}^{i} \frac{\partial V}{\partial q^{i}},
\end{aligned}
$$

where $\left(\mathcal{G}^{\mathcal{D}}\right)^{A B}$ denotes the coefficients of the inverse matrix of $\left(\mathcal{G}^{\mathcal{D}}\right)_{A B}$ with $\mathcal{G}^{\mathcal{D}}\left(e_{A}, e_{B}\right)=\left(\mathcal{G}^{\mathcal{D}}\right)_{A B}$.

Remark 3 Observe that these equations only depend on the adapted coordinates $\left(q^{i}, y^{A}\right)$ on $\mathcal{D}$. Therefore, the nonholonomic equations are free of Lagrange multipliers. These equations are equivalent to the nonholonomic Hamel equations (see, e.g., $[11,35]$ and the references therein).

\section{Optimal Control of Nonholonomic Mechanical Systems}

The purpose of this section is to study optimal control problems for nonholonomic mechanical systems. We assume that the controllability condition is satisfied, i.e., for any two points $q_{0}$ and $q_{f}$ in the configuration space $Q$, there exists an admissible control $u(t)$ defined on the control manifold $U \subseteq \mathbb{R}^{n}$, such that the system with initial condition $q_{0}$ reaches the point $q_{f}$ at time $T$ (see [4] and [13] for more details).

We will analyze the case when the dimension of the input or control distribution is equal to the rank of $\mathcal{D}$. If the rank of $\mathcal{D}$ is equal to the dimension of the control distribution, the system will be called a fully actuated nonholonomic system.

Definition 5 A solution of a fully actuated nonholonomic system is an admissible curve $\gamma: I \rightarrow \mathcal{D}$ such that

$$
\nabla_{\gamma(t)}^{\mathcal{G}^{\mathcal{D}}} \gamma(t)+\operatorname{grad}_{\mathcal{G}^{\mathcal{D}}} V\left(\tau_{\mathcal{D}}(\gamma(t))\right) \in \Gamma\left(\tau_{D}\right),
$$


or equivalently,

$$
\nabla_{\gamma(t)}^{\mathcal{G}^{\mathcal{D}}} \gamma(t)+\operatorname{grad}_{\mathcal{G}^{\mathcal{D}}} V\left(\tau_{\mathcal{D}}(\gamma(t))\right)=u^{A}(t) e_{A}\left(\tau_{\mathcal{D}}(\gamma(t))\right.
$$

where $u^{A}$ are the control inputs.

Locally, the equations may be written as follows

$$
\begin{aligned}
\dot{q}^{i} & =\rho_{A}^{i} y^{A}, \\
\dot{y}^{C} & =-\Gamma_{A B}^{C} y^{A} y^{B}-\left(\mathcal{G}^{\mathcal{D}}\right)^{C B} \rho_{B}^{i} \frac{\partial V}{\partial q^{i}}+u^{C} .
\end{aligned}
$$

Given a cost function

$$
\begin{aligned}
C: & \mathcal{D} \times U \rightarrow \mathbb{R} \\
& \left(q^{i}, y^{A}, u^{A}\right) \mapsto C\left(q^{i}, y^{A}, u^{A}\right),
\end{aligned}
$$

the optimal control problem consists of finding an admissible curve $\gamma: I \rightarrow \mathcal{D}$ solving the fully actuated nonholonomic problem, given initial and final boundary conditions on $\mathcal{D}$ and minimizing the functional

$$
\mathcal{\partial}(\gamma(t), u(t))=\int_{0}^{T} C(\gamma(t), u(t)) d t
$$

where $\gamma$ is an admissible curve.

Next, we define the submanifold $\mathcal{D}^{(2)}$ of $T \mathcal{D}$ by

$$
\mathcal{D}^{(2)}=\{v \in T \mathcal{D} \mid v=\dot{\gamma}(0) \text {, where } \gamma: I \rightarrow \mathcal{D} \text { is admissible }\},
$$

and we can choose coordinates $\left(x^{i}, y^{A}, \dot{y}^{A}\right)$ on $\mathcal{D}^{(2)}$, where the inclusion on $T \mathcal{D}$, denoted by $i_{\mathcal{D}^{(2)}}: \mathcal{D}^{(2)} \hookrightarrow T \mathcal{D}$ is given by

$$
i_{\mathcal{D}^{(2)}}\left(q^{i}, y^{A}, \dot{y}^{A}\right)=\left(q^{i}, y^{A}, \rho_{A}^{i}(q) y^{A}, \dot{y}^{A}\right) \text {. }
$$

Therefore, $\mathcal{D}^{(2)}$ is locally described by the constraints in $T \mathcal{D}$, given by

$$
\dot{q}^{i}-\rho_{A}^{i} y^{A}=0
$$

Observe now that our optimal control problem is alternatively determined by a smooth function $\mathcal{L}: \mathcal{D}^{(2)} \rightarrow \mathbb{R}$, where

$$
\mathcal{L}\left(q^{i}, y^{A}, \dot{y}^{C}\right)=C\left(q^{i}, y^{A}, \dot{y}^{C}+\Gamma_{A B}^{C} y^{A} y^{B}+\left(\mathcal{G}^{\mathcal{D}}\right)^{C B} \rho_{B}^{i} \frac{\partial V}{\partial q^{i}}\right) .
$$


The following diagram summarizes the situation

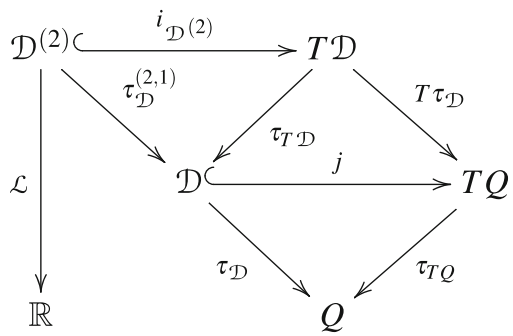

Here $j: \mathcal{D} \rightarrow T Q$ is the canonical inclusion from $\mathcal{D}$ to $T Q, \tau_{\mathcal{D}}^{(2,1)}: \mathcal{D}^{(2)} \rightarrow \mathcal{D}$ and $\tau_{T \mathcal{D}}: T \mathcal{D} \rightarrow \mathcal{D}$ are the projections locally given by $\tau_{\mathcal{D}}^{(2,1)}\left(q^{i}, y^{A}, \dot{y}^{A}\right)=\left(q^{i}, y^{A}\right)$ and $\tau_{T \mathcal{D}}\left(q^{i}, y^{A}, v^{i}, \dot{y}^{A}\right)=\left(q^{i}, y^{A}\right)$, respectively. Finally, $T \tau_{\mathcal{D}}: T \mathcal{D} \rightarrow T Q$ is locally described as follows: $\left(q^{i}, y^{A}, \dot{q}^{i}, \dot{y}^{A}\right) \mapsto\left(q^{i}, \dot{q}^{i}\right)$.

To derive the equations of motion, we use the standard variational calculus with constraints approach and, define the extended Lagrangian $\tilde{\mathcal{L}}: \mathcal{D}^{(2)} \times \mathbb{R}^{n} \rightarrow \mathbb{R}$, given as follows

$$
\tilde{\mathcal{L}}\left(q^{i}, y^{A}, \dot{y}^{A}, \lambda_{i}\right)=\mathcal{L}\left(q^{i}, y^{A}, \dot{y}^{A}\right)+\lambda_{i}\left(\dot{q}^{i}-\rho_{A}^{i} y^{A}\right) .
$$

Therefore, the equations of motion are

$$
\begin{aligned}
\frac{d}{d t}\left(\frac{\partial \tilde{\mathcal{L}}}{\partial \dot{q}^{i}}\right)-\frac{\partial \tilde{\mathcal{L}}}{\partial q^{i}} & =\dot{\lambda}_{i}-\frac{\partial \mathcal{L}}{\partial q^{i}}+\lambda_{j} \frac{\partial \rho_{A}^{j}}{\partial q^{i}} y^{A}=0, \\
\frac{d}{d t}\left(\frac{\partial \tilde{\mathcal{L}}}{\partial \dot{y}^{A}}\right)-\frac{\partial \tilde{\mathcal{L}}}{\partial y^{A}} & =\frac{d}{d t}\left(\frac{\partial \mathcal{L}}{\partial \dot{y}^{A}}\right)-\frac{\partial \mathcal{L}}{\partial y^{A}}+\rho_{A}^{i} \lambda_{i}=0, \\
\dot{q}^{i} & =\rho_{A}^{i} y^{A} .
\end{aligned}
$$

\subsection{Example: Continuously Variable Transmission (CVT)}

We want to study the optimal control of a simple model of a continuously variable transmission, where we assume that the belt cannot slip (see [36] for more details).

The shafts are connected to spiral springs fixed to the chasis. The belt between the two cones is translated along the shafts in accordance with the coordinate $x$, providing a varying transmission ratio. The belt moves in a plane perpendicular to the shafts, so that the belt has constant length (see [36] for a complete description and integrability of this system). The variables $\theta_{1}$ and $\theta_{2}$ denote the angular deflections of the shafts, $m$ is mass of the belt slider, $J_{1}>0$ is the inertia about the center of mass of the driving pulley and $J_{2}>0$ is the inertia about the center 
of mass of the driven pulley. The configuration space is $\mathbb{S}^{1} \times \mathbb{S}^{1} \times \mathbb{R}$ and the local coordinates on the configuration space are $q=\left(\theta_{1}, \theta_{2}, x\right) \in \mathbb{S}^{1} \times \mathbb{S}^{1} \times \mathbb{R}$.

The control inputs are $u_{1}$ and $u_{2}$. The first is a force applied perpendicular to the center of mass of the belt slider and the second is the torque applied about the center of mass of the driving pulley. We assume that $x<1$ (which corresponds to finite gear ratio).

The belt gives a constraint due to no slippage and is expressed in differential form by

$$
\omega=x d \theta_{1}-(1-x) d \theta_{2}
$$

Therefore the constraint distribution is given by $\mathcal{D}=\operatorname{span}\left\{X_{1}(q), X_{2}(q)\right\}$ where

$$
X_{1}(q)=\frac{1}{m} \frac{\partial}{\partial x} \text { and } X_{2}(q)=(1-x) \frac{\partial}{\partial \theta_{1}}+x \frac{\partial}{\partial \theta_{2}} \text {. }
$$

The Lagrangian is metric on $Q=\mathbb{S}^{1} \times \mathbb{S}^{1} \times \mathbb{R}$, where the matrix associated with the metric $\mathcal{G}$ is

$$
\mathcal{G}=\left(\begin{array}{ccc}
J_{1} & 0 & 0 \\
0 & J_{2} & 0 \\
0 & 0 & m
\end{array}\right)
$$

Then the Lagrangian $L: T\left(\mathbb{S}^{1} \times \mathbb{S}^{1} \times \mathbb{R}\right) \rightarrow \mathbb{R}$ is given by

$$
L(q, \dot{q})=\frac{1}{2}\left(J_{1} \dot{\theta}_{1}^{2}+J_{2} \dot{\theta}_{2}^{2}+m \dot{x}^{2}\right) .
$$

The projection map $\mathcal{P}: T\left(\mathbb{S}^{1} \times \mathbb{S}^{1} \times \mathbb{R}\right) \rightarrow \mathcal{D}$ is

$$
\begin{aligned}
& \mathcal{P}(q, \dot{q})=\frac{J_{1}(1-x)^{2}}{J_{1}-2 J_{1} x+J_{1} x^{2}+J_{2} x^{2}} d \theta_{1} \otimes \frac{\partial}{\partial \theta_{1}}+\frac{J_{1} x(1-x)}{J_{1}-2 J_{1} x+J_{1} x^{2}+J_{2} x^{2}} d \theta_{1} \otimes \frac{\partial}{\partial \theta_{2}} \\
& +\frac{J_{2} x(1-x)}{J_{1}-2 J_{1} x+J_{1} x^{2}+J_{2} x^{2}} d \theta_{2} \otimes \frac{\partial}{\partial \theta_{1}}+\frac{J_{2} x^{2}}{J_{1}-2 J_{1} x+J_{1} x^{2}+J_{2} x^{2}} d \theta_{2} \otimes \frac{\partial}{\partial \theta_{2}}+d x \otimes \frac{\partial}{\partial x} .
\end{aligned}
$$

Let $q=\left(\theta_{1}, \theta_{2}, x\right)$ be coordinates on the base manifold $Q=\mathbb{S}^{1} \times \mathbb{S}^{1} \times \mathbb{R}$ and take the basis $\left\{X_{1}, X_{2}\right\}$ of vector fields on $\mathcal{D}$. This basis induces adapted coordinates $\left(\theta_{1}, \theta_{2}, x, y_{1}, y_{2}\right) \in \mathcal{D}$ in the following way: Given the vector fields $X_{1}$ and $X_{2}$ generating the distribution $\mathcal{D}$, we obtain the relations for $q \in \mathbb{S}^{1} \times \mathbb{S}^{1} \times \mathbb{R}$ and

$$
X_{i}(q)=\rho_{i}^{1}(q) \frac{\partial}{\partial \theta_{1}}+\rho_{i}^{2}(q) \frac{\partial}{\partial \theta_{2}}+\rho_{i}^{3}(q) \frac{\partial}{\partial x}, \quad i=1,2 .
$$


Then,

$$
\rho_{1}^{1}=\rho_{1}^{2}=\rho_{2}^{3}=0, \quad \rho_{1}^{3}=\frac{1}{m}, \quad \rho_{2}^{1}=1-x, \quad \rho_{2}^{2}=x .
$$

Each element $e \in \mathcal{D}_{q}$ is expressed as a linear combination of these vector fields

$$
e=y_{1} X_{1}(q)+y_{2} X_{2}(q), \quad q \in \mathbb{S}^{1} \times \mathbb{S}^{1} \times \mathbb{R} .
$$

Therefore, the vector subbundle $\tau_{\mathcal{D}}: \mathcal{D} \rightarrow \mathbb{S}^{1} \times \mathbb{S}^{1} \times \mathbb{R}$ is locally described by the coordinates $\left(\theta_{1}, \theta_{2}, x, y_{1}, y_{2}\right)$; the first three for the base and the last two, for the fibers. Observe that

$$
e=y_{1}\left(\frac{1}{m} \frac{\partial}{\partial x}\right)+y_{2}\left((1-x) \frac{\partial}{\partial \theta_{1}}+x \frac{\partial}{\partial \theta_{2}}\right)
$$

and, as a consequence, $\mathcal{D}$ is described by the conditions (admissibility conditions)

$$
\dot{\theta}_{1}=(1-x) y_{2}, \quad \dot{\theta}_{2}=x y_{2}, \quad \dot{x}=\frac{1}{m} y_{1},
$$

as a vector subbundle of $T Q$, where $y_{1}$ and $y_{2}$ are the adapted velocities relative to the basis of $\mathcal{D}$ defined before. The nonholonomic bracket is given by $\llbracket \cdot, \cdot \rrbracket=\mathcal{P}([\cdot, \cdot])$. Observe now

$$
\begin{aligned}
\llbracket X_{1}, X_{2} \rrbracket=\mathcal{P}\left[X_{1}, X_{2}\right] & =\mathcal{P}\left(-\frac{1}{m} \frac{\partial}{\partial \theta_{1}}+\frac{1}{m} \frac{\partial}{\partial \theta_{2}}\right) \\
& =-\frac{1}{m} \frac{J_{1}(1-x)-J_{2} x}{J_{2} x^{2}+J_{1}(1-x)^{2}}\left((1-x) \frac{\partial}{\partial \theta_{1}}+x \frac{\partial}{\partial \theta_{2}}\right) .
\end{aligned}
$$

The restricted Lagrangian in these new adapted coordinates is rewritten as

$$
\ell\left(\theta_{1}, \theta_{2}, x, y_{1}, y_{2}\right)=\frac{y_{2}^{2}}{2}\left((1-x)^{2} J_{1}+J_{2} x^{2}\right)+\frac{1}{2 m} y_{1}^{2} .
$$

The Euler-Lagrange equations, together with the admissibility conditions for this Lagrangian are

$$
\frac{\dot{y}_{1}}{m}=0, \quad \dot{y}_{2} B(x)-\frac{y_{1} y_{2} A(x)}{m}=0, \quad \dot{\theta}_{1}=(1-x) y_{2}, \quad \dot{\theta}_{2}=x y_{2}, \quad \dot{x}=\frac{1}{m} y_{1},
$$

where $A(x)=J_{1}(1-x)-J_{2} x$ and $B(x)=(1-x)^{2} J_{1}+J_{2} x^{2}$. 
Next, we add controls in our picture. Therefore, the controlled Euler-Lagrange equations are

$$
u_{1}=\dot{y}_{2} B(x)-\frac{y_{1} y_{2} A(x)}{m}, \quad u_{2}=\frac{\dot{y}_{1}}{m}
$$

together with

$$
\dot{\theta}_{1}=(1-x) y_{2}, \quad \dot{\theta}_{2}=x y_{2}, \quad \dot{x}=\frac{1}{m} y_{1} .
$$

The optimal control problem consists of finding an admissible curve satisfying the previous equations, given boundary conditions on $\mathcal{D}$, and minimizing the functional

$$
\mathcal{J}\left(\theta_{1}, \theta_{2}, x, y_{1}, y_{2}, u_{1}, u_{2}\right)=\frac{1}{2} \int_{0}^{T}\left(u_{1}^{2}+u_{2}^{2}\right) d t,
$$

for the cost function $C: \mathcal{D} \times U \rightarrow \mathbb{R}$ given by

$$
C\left(\theta_{1}, \theta_{2}, x, y_{1}, y_{2}, u_{1}, u_{2}\right)=\frac{1}{2}\left(u_{1}^{2}+u_{2}^{2}\right)
$$

This optimal control problem is equivalent to the constrained variational problem determined by the lagrangian $\mathcal{L}: \mathcal{D}^{(2)} \rightarrow \mathbb{R}$, given by

$$
\mathcal{L}\left(\theta_{1}, \theta_{2}, x, y_{1}, y_{2}, \dot{y}_{1}, \dot{y}_{2}\right)=\frac{1}{2}\left(\dot{y}_{2} B(x)-\frac{y_{1} y_{2} A(x)}{m}\right)^{2}+\frac{\dot{y}_{1}^{2}}{2 m^{2}} .
$$

Here, $\mathcal{D}^{(2)}$ is a submanifold of the vector bundle $T \mathcal{D}$ over $\mathcal{D}$ defined by

$$
\begin{aligned}
\mathcal{D}^{(2)}= & \left\{\left(\theta_{1}, \theta_{2}, x, y_{1}, y_{2}, \dot{\theta}_{1}, \dot{\theta}_{2}, \dot{x}, \dot{y}_{1}, \dot{y}_{2}\right) \in T \mathcal{D} \mid \dot{x}-\frac{1}{m} y_{1}=0,\right. \\
& \left.\dot{\theta}_{1}-(1-x) y_{2}=0, \dot{\theta}_{2}-x y_{2}=0\right\},
\end{aligned}
$$

where $i_{\mathcal{D}^{(2)}}: \mathcal{D}^{(2)} \hookrightarrow T \mathcal{D}$, is given by the map

$$
i_{\mathcal{D}^{(2)}}\left(\theta_{1}, \theta_{2}, x, y_{1}, y_{2}, \dot{y}_{1}, \dot{y}_{2}\right)=\left(\theta_{1}, \theta_{2}, x, y_{1}, y_{2},(1-x) y_{2}, x y_{2}, \frac{y_{1}}{m}, \dot{y}_{1}, \dot{y}_{2}\right) \text {. }
$$

The equations of motion for the extended Lagrangian

$$
\begin{aligned}
& \tilde{\mathcal{L}}\left(\theta_{1}, \theta_{2}, x, y_{1}, y_{2}, \dot{\theta}_{1}, \dot{\theta}_{2}, \dot{x}, \dot{y}_{1}, \dot{y}_{2}, \lambda\right)= \\
& \mathcal{L}+\lambda_{1}\left(\dot{\theta}_{1}-(1-x) y_{2}\right)+\lambda_{2}\left(\dot{\theta}_{2}-x y_{2}\right)+\lambda_{3}\left(\dot{x}-\frac{1}{m} y_{1}\right),
\end{aligned}
$$


are

$$
\begin{aligned}
\dot{\lambda}_{1} & =0, \quad \dot{\lambda}_{2}=0, \\
\dot{\lambda}_{3} & =y_{2}\left(\lambda_{1}-\lambda_{2}\right)+\left(\dot{y}_{2} B(x)-A(x) \frac{y_{1} y_{2}}{m}\right)\left(\frac{y_{1} y_{2}\left(J_{1}+J_{2}\right)}{m}-2 \dot{y}_{2} A(x)\right), \\
\lambda_{3} & =-\frac{\ddot{y}_{1}}{m}-A y_{2}\left(\dot{y}_{2} B(x)-A(x) \frac{y_{1} y_{2}}{m}\right), \\
0 & =\lambda_{1}(1-x)+\lambda_{2} x-\frac{3}{m} y_{1} A(x)\left(\dot{y}_{2} B(x)-A(x) \frac{y_{1} y_{2}}{m}\right) \\
& +B(x)\left(\ddot{y}_{2} B(x)+\dot{y}_{2} \frac{2 y_{1} A(x)}{m}-\frac{1}{m}\left(A\left(\dot{y}_{1} y_{2}+\dot{y}_{2} y_{1}\right)-\frac{y_{1}^{2}\left(J_{1}+J_{2}\right)}{m}\right)\right),
\end{aligned}
$$

with

$$
\dot{\theta}_{1}=(1-x) y_{2}, \quad \dot{\theta}_{2}=x y_{2}, \quad \dot{x}=\frac{1}{m} y_{1} .
$$

The resulting system of equations for the optimal control problem of the continuously variable transmission is difficult to solve explicitly and from this observation, it is clear that it is necessary to develop numerical methods, which preserve the geometric structure for these mechanical control systems. The construction of geometric numerical methods for this kind of optimal control problem is a topic future of research, as we remark in Sect. 5.

\subsection{Example: Chaplygin Sleigh}

We want to study the optimal control of the so-called Chaplygin sleigh (see [4]) introduced and studied in 1911 by Chaplygin (see [14] and [38]). The sleigh is a rigid body moving on a horizontal plane supported at three points, two of which slide freely without friction while the third is a knife edge which allows no motion in the direction orthogonal to the sleigh as shown in Fig. 1.

We assume that the sleigh cannot move sideways. The configuration space of this dynamical system is the special Euclidean group $S E(2)$, with local coordinates $q=(x, y, \theta)$, since an element $A \in \operatorname{SE}(2)$ is represented by the matrix

$$
A=\left(\begin{array}{ccc}
\cos \theta & -\sin \theta & x \\
\sin \theta & \cos \theta & y \\
0 & 0 & 1
\end{array}\right),
$$

where $\theta \in \mathbb{S}^{1}$ and $(x, y) \in R^{2}$ are the angular orientation of the sleigh and position of the contact point of the sleigh on the plane, respectively. Let $m$ be the mass of the 


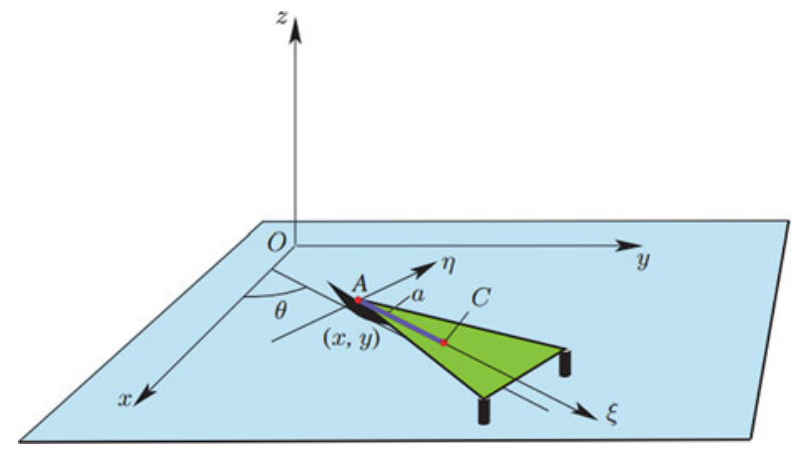

Fig. 1 The Chaplygin sleigh

sleigh and $I+m a^{2}$ be the inertia about the contact point, where $I$ is the moment of inertia about the center of mass $C$ and $a$ is the distance from the center of mass to the knife edge.

The control inputs are denoted by $u_{1}$ and $u_{2}$. The first one corresponds to a force applied perpendicular to the center of mass of the sleigh and the second one is the torque applied about the vertical axis.

The constraint is given by the no slip condition and is expressed in differential form by

$$
\omega=\sin \theta d x-\cos \theta d y .
$$

Therefore the constraint distribution is given by $\mathcal{D}=\operatorname{span}\left\{X_{1}(q), X_{2}(q)\right\}$ where

$$
X_{1}(q)=\frac{1}{J} \frac{\partial}{\partial \theta} \text { and } X_{2}(q)=\frac{\cos \theta}{m} \frac{\partial}{\partial x}+\frac{\sin \theta}{m} \frac{\partial}{\partial y} \text {. }
$$

The Lagrangian is metric on $S E(2)$, where the matrix associated with the metric $\mathcal{G}$ is

$$
\mathcal{G}=\left(\begin{array}{ccc}
m & 0 & 0 \\
0 & m & 0 \\
0 & 0 & J
\end{array}\right)
$$

The Lagrangian $L: T\left(\mathbb{R}^{2} \times \mathbb{S}^{1}\right) \rightarrow \mathbb{R}$ is given by the kinetic energy of the body, which is a sum of the translational kinetic energy and the rotational kinetic energy of the body

$$
L(q, \dot{q})=\frac{m}{2}\left(\dot{x}_{C}^{2}+\dot{y}_{C}^{2}\right)+\frac{J}{2} \dot{\theta}^{2},
$$


where $x_{C}=x+a \cos \theta$ and $y_{C}=y+a \sin \theta$. The projection map $\mathcal{P}: T Q \rightarrow \mathcal{D}$ is

$$
\begin{aligned}
\mathcal{P}(q, \dot{q})= & \cos ^{2} \theta d x \otimes \frac{\partial}{\partial x}+\cos \theta \sin \theta d x \otimes \frac{\partial}{\partial y}+\cos \theta \sin \theta d y \otimes \frac{\partial}{\partial x} \\
& +\sin ^{2} \theta d y \otimes \frac{\partial}{\partial y}+d \theta \otimes \frac{\partial}{\partial \theta} .
\end{aligned}
$$

Let $q=(x, y, \theta)$ be coordinates on the base manifold $\mathbb{R}^{2} \times \mathbb{S}^{1}$ and take the basis $\left\{X_{1}, X_{2}\right\}$ of vector fields of $\mathcal{D}$. This basis induces adapted coordinates $\left(x, y, \theta, y_{1}, y_{2}\right) \in \mathcal{D}$ in the following way: Given the vector fields $X_{1}$ and $X_{2}$ generating the distribution, we obtain the relations for $q \in \mathbb{R}^{2} \times \mathbb{S}^{1}$

$$
X_{i}(q)=\rho_{i}^{1}(q) \frac{\partial}{\partial x}+\rho_{i}^{2}(q) \frac{\partial}{\partial y}+\rho_{i}^{3}(q) \frac{\partial}{\partial \theta}, \quad i=1,2 .
$$

Then,

$$
\rho_{1}^{1}=\rho_{1}^{2}=\rho_{2}^{3}=0, \quad \rho_{1}^{3}=\frac{1}{J}, \quad \rho_{2}^{1}=\frac{\cos \theta}{m}, \quad \rho_{2}^{2}=\frac{\sin \theta}{m} .
$$

Each element $e \in \mathcal{D}_{q}$ is expressed as a linear combination of these vector fields

$$
e=y_{1} X_{1}(q)+y_{2} X_{2}(q), \quad q \in \mathbb{R}^{2} \times \mathbb{S}^{1} .
$$

Therefore, the vector subbundle $\tau_{\mathcal{D}}: \mathcal{D} \rightarrow \mathbb{R}^{2} \times \mathbb{S}^{1}$ is locally described by the coordinates $\left(x, y, \theta, y_{1}, y_{2}\right)$; the first three for the base and the last two, for the fibers. Observe that

$$
e=y_{1}\left(\frac{1}{J} \frac{\partial}{\partial \theta}\right)+y_{2}\left(\frac{\cos \theta}{m} \frac{\partial}{\partial x}+\frac{\sin \theta}{m} \frac{\partial}{\partial y}\right)
$$

and, as a consequence, $\mathcal{D}$ is described by the conditions (admissibility conditions)

$$
\dot{x}=\frac{\cos \theta}{m} y_{2}, \quad \dot{y}=\frac{\sin \theta}{m} y_{2}, \quad \dot{\theta}=\frac{1}{J} y_{1}
$$

as a vector subbundle of $T Q$, where $y_{1}$ and $y_{2}$ are the adapted velocities relative to the basis of $\mathcal{D}$ defined before. The nonholonomic bracket given by $\llbracket \cdot, \cdot \rrbracket=\mathcal{P}([\cdot, \cdot])$ satisfies

$$
\llbracket X_{1}, X_{2} \rrbracket=\mathcal{P}\left[X_{1}, X_{2}\right]=\mathcal{P}\left(-\frac{1}{J m} \sin \theta \frac{\partial}{\partial x}+\frac{\cos \theta}{J m} \frac{\partial}{\partial y}\right)=0 .
$$


The restricted Lagrangian in the new adapted coordinates is given by

$$
\ell\left(x, y, \theta, y_{1}, y_{2}\right)=\frac{1}{2 m}\left(y_{2}\right)^{2}+\frac{b}{2 J}\left(y_{1}\right)^{2} \text { where } b=\frac{a^{2} m}{J} .
$$

Therefore, the equations of motion are

$$
\frac{b \dot{y}_{1}}{J}=0, \quad \frac{\dot{y}_{2}}{m}=0, \quad \dot{x}=\frac{\cos \theta}{m} y_{2}, \quad \dot{y}=\frac{\sin \theta}{m} y_{2}, \quad \dot{\theta}=\frac{1}{J} y_{1} .
$$

The controlled Euler-Lagrange equations are written as

$$
\frac{b \dot{y}_{1}}{J}=u_{2}, \quad \frac{\dot{y}_{2}}{m}=u_{1}, \quad \dot{x}=\frac{\cos \theta}{m} y_{2}, \quad \dot{y}=\frac{\sin \theta}{m} y_{2}, \quad \dot{\theta}=\frac{1}{J} y_{1} .
$$

The optimal control problem consists on finding an admissible curve satisfying the previous equations, given boundary conditions on $\mathcal{D}$, and minimizing the functional

$$
\mathcal{J}\left(x, y, \theta, y_{1}, y_{2}, u_{1}, u_{2}\right)=\frac{1}{2} \int_{0}^{T}\left(u_{1}^{2}+u_{2}^{2}\right) d t,
$$

for the cost function $C: \mathcal{D} \times U \rightarrow \mathbb{R}$ given by

$$
C\left(x, y, \theta, y_{1}, y_{2}, u_{1}, u_{2}\right)=\frac{1}{2}\left(u_{1}^{2}+u_{2}^{2}\right) .
$$

As before, the optimal control problem is equivalent to solving the constrained variational problem determined by $\mathcal{L}: \mathcal{D}^{(2)} \rightarrow \mathbb{R}$, given by

$$
\mathcal{L}\left(x, y, \theta, y_{1}, y_{2}, \dot{y}_{1}, \dot{y}_{2}\right)=\frac{1}{2}\left(\frac{b^{2} \dot{y}_{1}^{2}}{J^{2}}+\frac{\dot{y}_{2}^{2}}{m^{2}}\right) .
$$

Here, $\mathcal{D}^{(2)}$ is a submanifold of the vector bundle $T \mathcal{D}$ over $\mathcal{D}$ defined by

$$
\begin{aligned}
\mathcal{D}^{(2)}= & \left\{\left(x, y, \theta, y_{1}, y_{2}, \dot{x}, \dot{y}, \dot{\theta}, \dot{y}_{1}, \dot{y}_{2}\right) \in T \mathcal{D} \mid \dot{x}-\frac{\cos \theta}{m} y_{2}=0,\right. \\
& \left.\dot{y}-\frac{\sin \theta}{m} y_{2}=0, \dot{\theta}-\frac{1}{J} y_{1}=0\right\},
\end{aligned}
$$

where $i_{\mathcal{D}^{(2)}}: \mathcal{D}^{(2)} \hookrightarrow T \mathcal{D}$, is given by the map

$$
i_{\mathcal{D}^{(2)}}\left(x, y, \theta, y_{1}, y_{2}, \dot{y}_{1}, \dot{y}_{2}\right)=\left(x, y, \theta, y_{1}, y_{2}, \frac{\cos \theta}{m} y_{2}, \frac{\sin \theta}{m} y_{2}, \frac{1}{J} y_{1}, \dot{y}_{1}, \dot{y}_{2}\right) .
$$


The equations of motion for the extended Lagrangian

$$
\begin{aligned}
\tilde{\mathcal{L}}\left(x, y, \theta, y_{1}, y_{2}, \dot{x}, \dot{y}, \dot{\theta}, \dot{y}_{1}, \dot{y}_{2}, \lambda\right)= & \mathcal{L}+\lambda_{1}\left(\dot{x}-\frac{\cos \theta}{m} y_{2}\right)+\lambda_{2}\left(\dot{y}-\frac{\sin \theta}{m} y_{2}\right) \\
& +\lambda_{3}\left(\dot{\theta}-\frac{1}{J} y_{1}\right),
\end{aligned}
$$

with $\lambda=\left(\lambda_{1}, \lambda_{2}, \lambda_{3}\right) \in \mathbb{R}^{3}$, are

$$
\begin{aligned}
& \dot{\lambda}_{1}=0, \quad \dot{\lambda}_{2}=0, \quad \dot{\lambda}_{3}=\frac{y_{2}}{m}\left(\lambda_{1} \sin \theta-\lambda_{2} \cos \theta\right), \\
& \lambda_{3}=-\frac{b^{2} \ddot{y}_{1}}{J}, \quad \ddot{y}_{2}=-m\left(\lambda_{1} \cos \theta+\lambda_{2} \sin \theta\right),
\end{aligned}
$$

with

$$
\dot{x}=\frac{\cos \theta}{m} y_{2}, \quad \dot{y}=\frac{\sin \theta}{m} y_{2}, \quad \dot{\theta}=\frac{1}{J} y_{1} .
$$

The first two equations can be integrated and give $\lambda_{1}=c_{1}$ and $\lambda_{2}=c_{2}$, where $c_{1}$ and $c_{2}$ are constants and differentiating the equation for $\lambda_{3}$ with respect to the time and substituting into the third equation, the above equations reduce to the following equations

$$
\frac{\dddot{y}_{1}}{J}=\frac{y_{2}}{m b^{2}}\left(c_{2} \cos \theta-c_{1} \sin \theta\right), \quad \ddot{y}_{2}=-m\left(c_{1} \cos \theta+c_{2} \sin \theta\right),
$$

with

$$
\dot{x}=\frac{\cos \theta}{m} y_{2}, \quad \dot{y}=\frac{\sin \theta}{m} y_{2}, \quad \dot{\theta}=\frac{1}{J} y_{1} .
$$

If we suppose, $\lambda_{1}=0$ and $\lambda_{2}=0$ (that is, $c_{1}=c_{2}=0$ ) the first two equations above reduce to the following equations

$$
\dddot{y}_{1}=0 \text { and } \ddot{y}_{2}=0 .
$$

Integrating these equations and using the admissibility conditions, we obtain constants of integration $c_{i}, i=3, \ldots, 8$ and the equations

$$
\begin{aligned}
& \theta(t)=\frac{c_{3} t^{3}}{6 J}+\frac{c_{4} t^{2}}{2 J}+\frac{c_{5} t+c_{6}}{J}, \\
& x(t)=\frac{1}{m} \int_{0}^{t} \cos \left(\frac{c_{3} s^{3}+3 c_{4} s^{2}+6 c_{5} s+6 c_{6}}{6 J}\right)\left(c_{7} s+c_{8}\right) d s, \\
& y(t)=\frac{1}{m} \int_{0}^{t} \sin \left(\frac{c_{3} s^{3}+3 c_{4} s^{2}+6 c_{5} s+6 c_{6}}{6 J}\right)\left(c_{7} s+c_{8}\right) d s .
\end{aligned}
$$


Therefore, the controls $u_{1}$ and $u_{2}$ are

$$
u_{1}(t)=\frac{c_{7}}{m}, \quad u_{2}(t)=\frac{c_{3} t+c_{4}}{J} .
$$

Remark 4 A similar optimal control problem was studied also by Hussein and Bloch in [9]. The authors also used the theory of affine connections to analyze the optimal control problem of underactuated nonholonomic mechanical systems. The main difference with our approach is that in our paper we are working on the distribution $\mathcal{D}$ itself. We impose the extra condition $\lambda_{1}=\lambda_{2}=0$ to obtain explicitlly the controls minimizing the cost function. Usually, there are prescribed initial and final boundary conditions on $\mathcal{D}$. For the Chaplygin sleigh we impose conditions $\left(x(0), y(0), \theta(0), y_{1}(0), y_{2}(0)\right)$ and $\left(x(T), y(T), \theta(T), y_{1}(T), y_{2}(T)\right)$. Observe that if we transform these conditions into initial conditions we will need to take the initial condition $\left(x(0), y(0), \theta(0), y_{1}(0), y_{2}(0), \dot{y}_{1}(0), \dot{y}_{2}(0), \lambda_{1}(0), \lambda_{2}(0), \lambda_{3}(0)\right)$ and it is not necessary that some of the multipliers are zero from the very beginning.

\subsection{Application to Motion Planning for Obstacle Avoidance: Chaplygin Sleigh with Obstacles}

In this section, we use the model of the Chaplygin sleigh from the previous section to show how obstacle avoidance can be achieved with our approach using navigation functions. A navigation function is a potential field-based function used to model an obstacle as a repulsive area or surface (see [23, 24]).

For the Chaplygin sleigh, consider the following boundary conditions on the distribution $\mathcal{D}: x(0)=0, \quad y(1)=0, \quad \theta(0)=0, \quad y_{1}(0)=0, \quad y_{2}(0)=0$ and $x(T)=1, \quad y(T)=1, \quad \theta(T)=0, \quad y_{1}(T)=0, \quad y_{2}(T)=0$.

Let the obstacle be circular in shape, with its center located at the point $\left(x_{C}, y_{C}\right)=(0.5,0.5)$ in the $x y$-plane. For llustrative purposes, we use a simple inverse square law for the navigation function. Let $V(x, y)$ be given by

$$
V(x, y)=\frac{\kappa}{\left(x-x_{C}\right)^{2}+\left(y-y_{C}\right)^{2}},
$$

where the parameter $\kappa$ is introduced to control the strength of the potential function.

Appending the potential into the cost functional (5), the optimal control problem is equivalent to solving the constrained variational problem determined by $\mathcal{L}$ : $\mathcal{D}^{(2)} \rightarrow \mathbb{R}$, given by

$$
\mathcal{L}\left(x, y, \theta, y_{1}, y_{2}, \dot{y}_{1}, \dot{y}_{2}\right)=\frac{b^{2} \dot{y}_{1}^{2}}{2 J^{2}}+\frac{\dot{y}_{2}^{2}}{2 m^{2}}+\frac{\kappa}{2\left(\left(x-x_{C}\right)^{2}+\left(y-y_{C}\right)^{2}\right)} .
$$


The equations of motion for the extended Lagrangian

$$
\begin{aligned}
& \tilde{\mathcal{L}}\left(x, y, \theta, y_{1}, y_{2}, \dot{x}, \dot{y}, \dot{\theta}, \dot{y}_{1}, \dot{y}_{2}, \lambda\right)= \mathcal{L} \\
&+\lambda_{1}\left(\dot{x}-\frac{\cos \theta}{m} y_{2}\right)+\lambda_{2}\left(\dot{y}-\frac{\sin \theta}{m} y_{2}\right) \\
&+\lambda_{3}\left(\dot{\theta}-\frac{1}{J} y_{1}\right)
\end{aligned}
$$

with $\lambda=\left(\lambda_{1}, \lambda_{2}, \lambda_{3}\right) \in \mathbb{R}^{3}$ are

$$
\begin{aligned}
& \dot{\lambda}_{1}=-\frac{\kappa\left(x-x_{C}\right)}{\left(\left(x-x_{C}\right)^{2}+\left(y-y_{C}\right)^{2}\right)^{2}} \quad \dot{\lambda}_{2}=-\frac{\kappa\left(y-y_{C}\right)}{\left(\left(x-x_{C}\right)^{2}+\left(y-y_{C}\right)^{2}\right)^{2}}, \\
& \dot{\lambda}_{3}=\frac{y_{2}}{m}\left(\lambda_{1} \sin \theta-\lambda_{2} \cos \theta\right), \quad \lambda_{3}=-\frac{b^{2} \ddot{y}_{1}}{J}, \quad \ddot{y}_{2}=-m\left(\lambda_{1} \cos \theta+\lambda_{2} \sin \theta\right),
\end{aligned}
$$

with

$$
\dot{x}=\frac{\cos \theta}{m} y_{2}, \quad \dot{y}=\frac{\sin \theta}{m} y_{2}, \quad \dot{\theta}=\frac{1}{J} y_{1} .
$$

We solve the earlier boundary value problem for several values of $\kappa$. Starting with $\kappa=0$, which corresponds to a zero potential function, we increment $\kappa$ until the potential field was strong enough to prevent the sleigh from interfering with the obstacle. We took $\kappa=0,0.01,0.1,0.25$, and 0.5 and $T=1$. The result is shown in Fig. 2. Note that for $\kappa=0.25$ and 0.5 the sleigh avoids the obstacle and as one may anticipate, as $\kappa$ increases, the total control effort and therefore, the total $\operatorname{cost} \mathcal{J}=\frac{1}{2} \int_{0}^{1}\left(u_{1}^{2}+u_{2}^{2}+V(x, y)\right) d t$ increases. For example, $\mathcal{J}=17.0242$, when $\kappa=0.25$ and $\mathcal{J}=18.4634$, when $\kappa=0.5$. Hence, we select $\kappa=0.25$ since it
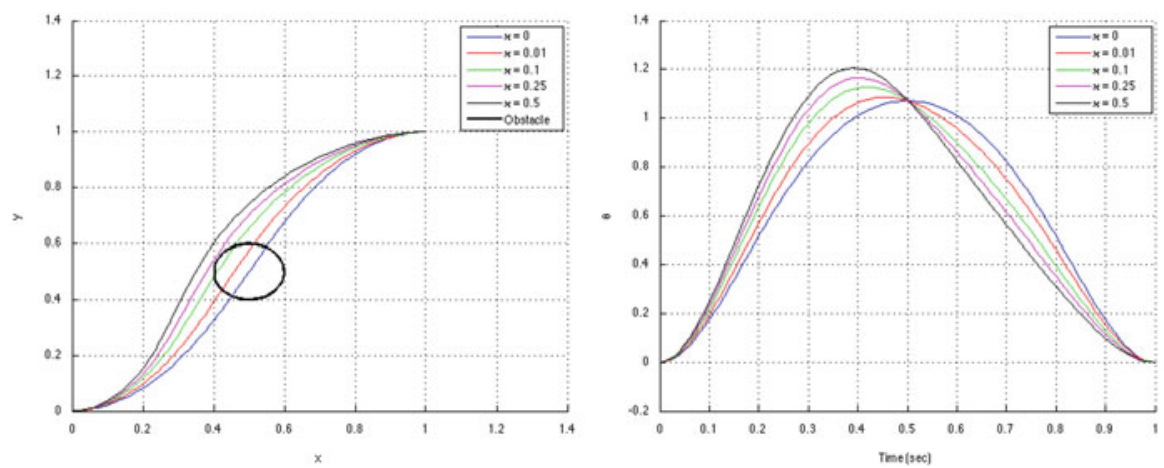

Fig. 2 The extremals solving the boundary value problem (left) and behavior of $\theta$ (right) for $\kappa=0,0.01,0.1,0.25$ and 0.5 

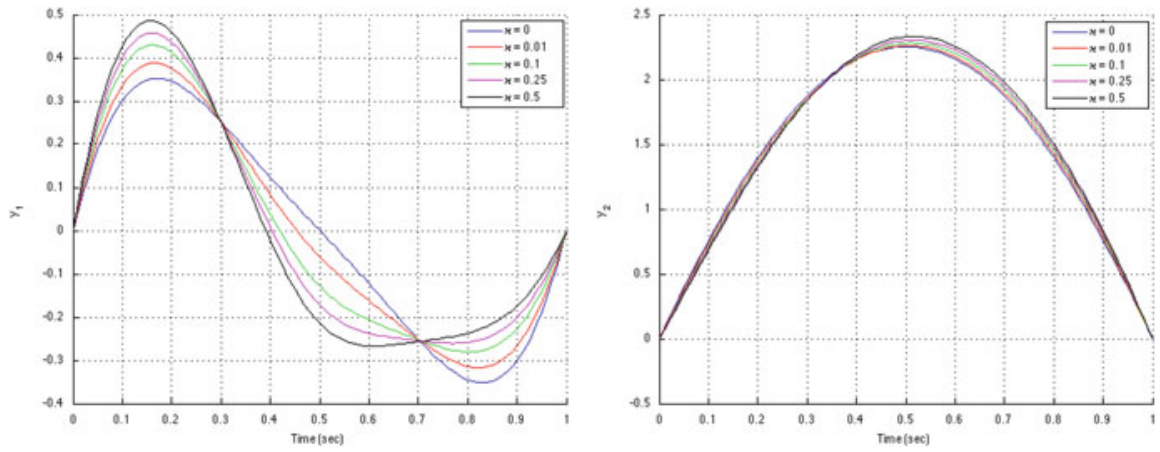

Fig. 3 Behavior of the velocites $y_{1}$ (left) and $y_{2}$ (right) for $\kappa=0,0.01,0.1,0.25$ and 0.5
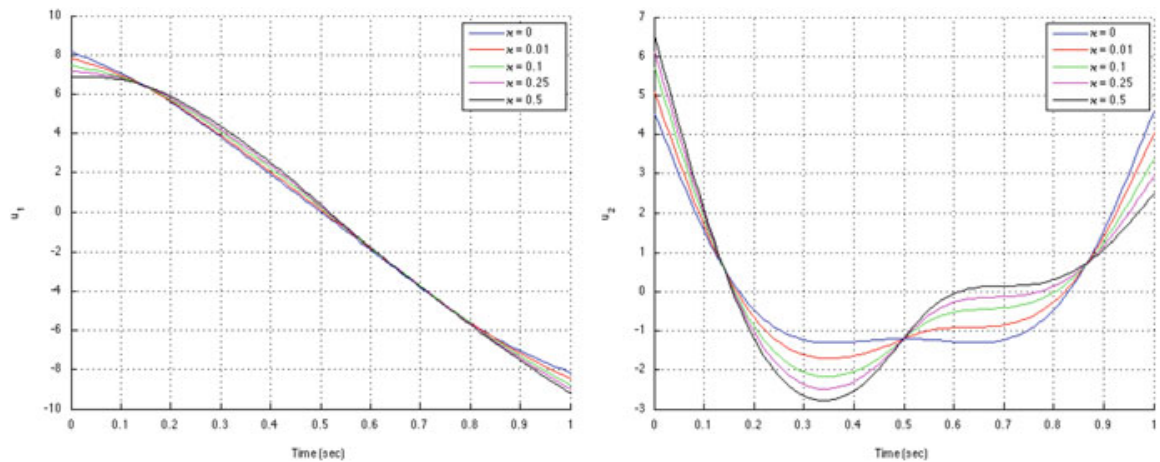

Fig. 4 Behavior of the controls $u_{1}($ left $)$ and $u_{2}$ (right) for $\kappa=0,0.01,0.1,0.25$ and 0.5

corresponds to a trajectory that avoids the obstacle with the least possible cost (of all five values of $\kappa$ tried in this simulation). The trajectories on $\mathcal{D}$ and the control inputs $u_{1}$ and $u_{2}$ for the different values of $\kappa$ are shown in Figs. 3, 4. This example illustrates how our approach can be used with the method of navigation functions for optimal control purposes with obstacle avoidance.

\section{Lagrangian Submanifolds and Nonholonomic Optimal Control Problems}

In this section we study the construction of a Lagrangian submanifold representing intrinsically the dynamics of the optimal control problem and the corresponding Hamiltonian representation when the system is regular. In the regular case, the definition of a particular Legendre transformation gives rise to the relationship and correspondence between the Lagrangian and Hamiltonian dynamics. 


\subsection{Lagrangian Submanifolds}

In this subsection we will construct Lagrangian submanifolds that are interesting for our purposes in the study of the geometry of optimal control problems of controlled mechanical systems (see [33] and [46]).

Definition 6 Given a finite-dimensional symplectic manifold $(P, \omega)$ and a submanifold $N$, with canonical inclusion $i_{N}: N \hookrightarrow P, N$ is said to be a Lagrangian submanifold if $i_{N}^{*} \omega=0$ and $\operatorname{dim} N=\frac{1}{2} \operatorname{dim} P$.

A distinguished symplectic manifold is the cotangent bundle $T^{*} Q$ of any manifold $Q$. If we choose local coordinates $\left(q^{i}\right), 1 \leq i \leq n$, then $T^{*} Q$ has induced coordinates $\left(q^{i}, p_{i}\right)$. Denote by $\pi_{Q}: T^{*} Q \rightarrow Q$ the canonical projection of the cotangent bundle given by $\pi_{Q}\left(\epsilon_{q}\right)=q$, where $\epsilon_{q} \in T_{q}^{*} Q$. Define the Liouville 1 -form or canonical the 1 -form $\theta_{Q} \in \Lambda^{1}\left(T^{*} Q\right)$ by

$$
\left\langle\left(\theta_{Q}\right)_{\epsilon}, X\right\rangle=\left\langle\epsilon, T \pi_{Q}(X)\right\rangle \text {, where } X \in T_{\epsilon} T^{*} Q, \epsilon \in T^{*} Q \text {. }
$$

In local coordinates we have that $\theta_{Q}=p_{i} \mathrm{~d} q^{i}$. The canonical two-form $\omega_{Q}$ on $T^{*} Q$ is the symplectic form $\omega_{Q}=-\mathrm{d} \theta_{Q}$ (i.e., $\omega_{Q}=\mathrm{d} q^{i} \wedge \mathrm{d} p_{i}$ ).

Now, we will introduce some special Lagrangian submanifolds of the symplectic manifold $\left(T^{*} Q, \omega_{Q}\right)$. For instance, the image $\Sigma_{\lambda}=\lambda(Q) \subset T^{*} Q$ of a closed 1-form $\lambda \in \Lambda^{1} Q$ is a Lagrangian submanifold of $\left(T^{*} Q, \omega_{Q}\right)$, since $\lambda^{*} \omega_{Q}=-d \lambda=0$. We then obtain a submanifold diffeomorphic to $Q$ and transverse to the fibers of $T^{*} Q$. When $\lambda$ is exact, i.e., $\lambda=\mathrm{d} f$, where $f: Q \rightarrow \mathbb{R}$, we say that $f$ is a generating function of the Lagrangian submanifold $\Sigma_{\lambda}=\Sigma_{f}$ (see [46]).

A useful extension of the previous construction is the following: given a manifold $Q$ and a function $S: Q \rightarrow \mathbb{R}$, the submanifold $\mathrm{d} S \subset T^{*} Q$ is Lagrangian. There is a more general construction given by Śniatycki and Tulczyjew [41] (see also [44] and [45]), which we will use to generate the dynamics.

Theorem 1 (Śniatycki and Tulczyjew [41]) Let $Q$ be a smooth manifold, $N \subset Q$ a submanifold, and $S: N \rightarrow \mathbb{R}$. Then

$$
\begin{aligned}
& \Sigma_{S}=\left\{\mu \in T^{*} Q \mid \pi_{Q}(\mu) \in N \text { and }\langle\mu, v\rangle=\langle\mathrm{d} S, v\rangle\right. \\
& \text { for all } \left.v \in T N \subset T Q \text { such that } \tau_{Q}(v)=\pi_{Q}(\mu)\right\}
\end{aligned}
$$

is a Lagrangian submanifold of $T^{*} Q$. Here $\pi_{Q}: T^{*} Q \rightarrow Q$ and $\tau_{Q}: T Q \rightarrow Q$ denote the cotangent and tangent bundle projections, respectively.

Taking $f$ as the zero function, for example, we obtain the following Lagrangian submanifold

$$
\Sigma_{0}=\left\{\left.p \in T^{*} Q\right|_{N} \mid\langle p, v\rangle=0, \forall v \in T N \text { with } \tau_{Q}(v)=\pi_{Q}(p)\right\},
$$


which is just the conormal bundle of $N$ :

$$
v^{*}(N)=\left\{\left.p \in T^{*} Q\right|_{N} \text { such that }\left.p\right|_{T_{\pi(p)} N}=0\right\} .
$$

\subsection{Lagrangian Submanifold Description of Nonholonomic Mechanical Control Problems}

Next, we derive the equations of motion representing the dynamics of the optimal control problem.

Given the function $\mathcal{L}: \mathcal{D}^{(2)} \rightarrow \mathbb{R}$, following Theorem 1 , when $N=\mathcal{D}^{(2)} \subset$ $T \mathcal{D}$ we have the Lagrangian submanifold $\Sigma_{\mathcal{L}} \subset T^{*} T \mathcal{D}$. Therefore, $\mathcal{L}: \mathcal{D}^{(2)} \rightarrow$ $\mathbb{R}$ generates a Lagrangian submanifold $\Sigma_{\mathcal{L}} \subset T^{*} T \mathcal{D}$ of the symplectic manifold $\left(T^{*} T \mathcal{D}, \omega_{T \mathcal{D}}\right)$, where $\omega_{T \mathcal{D}}$ is the canonical symplectic 2 -form on $T^{*} T \mathcal{D}$.

The relationship between these spaces is summarized in the following diagram

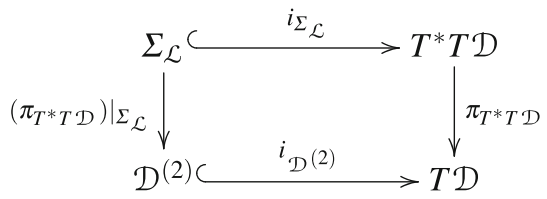

Proposition 1 Let $\mathcal{L}: \mathcal{D}^{(2)} \rightarrow \mathbb{R}$ be a $C^{\infty}$-function. Consider the inclusion $i_{\mathcal{D}^{(2)}}$ : $\mathcal{D}^{(2)} \rightarrow T \mathcal{D}$, where $\omega_{T \mathcal{D}}$ is the canonical symplectic 2 -form in $T^{*} T \mathcal{D}$. Then

$$
\Sigma_{\mathcal{L}}=\left\{\mu \in T^{*} T \mathcal{D} \mid i_{\Sigma_{\mathcal{L}}}^{*} \mu=d \mathcal{L}\right\} \subset T^{*} T \mathcal{D}
$$

is a Lagrangian submanifold of $\left(T^{*} T \mathcal{D}, \omega_{T \mathcal{D}}\right)$.

Definition 7 Let $\mathcal{D}$ be a non-integrable distribution, $T \mathcal{D}$ its tangent bundle and $\mathcal{D}^{(2)}$ the subbundle of $T \mathcal{D}$ defined in (2). A second-order nonholonomic system is a pair $\left(\mathcal{D}^{(2)}, \Sigma_{\mathcal{L}}\right.$ ), where $\Sigma_{\mathcal{L}} \subset T^{*} T \mathcal{D}$ is the Lagrangian submanifold generated by $\mathcal{L}$ : $\mathcal{D}^{(2)} \rightarrow \mathbb{R}$.

Consider local coordinates $\left(q^{i}, y^{A}, \dot{q}^{i}, \dot{y}^{A}\right)$ on $T \mathcal{D}$. These coordinates induce local coordinates $\left(q^{i}, y^{A}, \dot{q}^{i}, \dot{y}^{A}, \mu_{i}, \mu_{A}, \gamma_{i}, \gamma_{A}\right)$ on $T^{*} T \mathcal{D}$. Therefore, locally, the system is characterized by the following set of equations on $T^{*} T \mathcal{D}$

$$
\begin{aligned}
\mu_{i}+\gamma_{j} \frac{\partial \rho_{A}^{j}}{\partial q^{i}} y^{A} & =\frac{\partial \mathcal{L}}{\partial q^{i}}, \\
\mu_{A}+\gamma_{j} \rho_{A}^{j} & =\frac{\partial \mathcal{L}}{\partial y^{A}},
\end{aligned}
$$




$$
\begin{aligned}
& \gamma_{A}=\frac{\partial \mathcal{L}}{\partial \dot{y}^{A}}, \\
& \dot{q}^{i}=\rho_{A}^{i} y^{A} .
\end{aligned}
$$

Remark 5 Typically, local coordinates on $\Sigma_{\mathcal{L}} \subset T^{*} T \mathcal{D}$ are $\left(q^{i}, y^{A}, \dot{y}^{A}, \gamma_{i}\right)$, where $\gamma_{i}$ plays the role of the Lagrange multipliers.

Remark 6 In the case of the Chaplygin sleigh, local coordinates on $T^{*} T \mathcal{D}$ will be given by $\left(x, y, \theta, y_{1}, y_{2}, \dot{x}, \dot{y}, \dot{\theta}, \dot{y}_{1}, \dot{y}_{2}, \mu_{x}, \mu_{y}, \mu_{\theta}, \mu_{1}, \mu_{2}, \gamma_{x}, \gamma_{y}, \gamma_{\theta}, \gamma_{1}, \gamma_{2}\right)$, where the local coordinates on $T \mathcal{D}$ are $\left(x, y, \theta, y_{1}, y_{2}, \dot{x}, \dot{y}, \dot{\theta}, \dot{y}_{1}, \dot{y}_{2}\right)$. The Lagrangian submanifold of $T^{*} T \mathcal{D}$ is described by the equations

$$
\begin{aligned}
\mu_{x} & =0, \quad \mu_{y}=0, \\
\mu_{\theta} & =\frac{y_{2}}{m}\left(\gamma_{x} \sin \theta-\gamma_{y} \cos \theta\right), \\
\mu_{1} & =-\frac{b^{2} \gamma_{\theta}}{J}, \quad \mu_{2}=-m\left(\gamma_{x} \cos \theta+\gamma_{y} \cos \theta\right), \\
\gamma_{1} & =\frac{b^{2} \dot{y}_{1}}{J^{2}}, \quad \gamma_{2}=\frac{\dot{y}_{2}}{m^{2}}, \\
\dot{x} & =\frac{\cos \theta}{m} y_{2}, \quad \dot{y}=\frac{\sin \theta}{m} y_{2}, \quad \dot{\theta}=\frac{y_{1}}{J} .
\end{aligned}
$$

After a straightforward computation one can check easily that these equations are equivalent to those obtained in the Lagrangian formalism.

\subsection{Legendre Transformation and Regularity Condition}

We define the map $\Psi: T^{*} T \mathcal{D} \rightarrow T^{*} \mathcal{D}$ as

$$
\left\langle\Psi\left(\mu_{v_{x}}\right), X(x)\right\rangle=\left\langle\mu_{v_{x}}, X^{V}\left(v_{x}\right)\right\rangle,
$$

where $\mu \in T^{*} T \mathcal{D}, v_{x} \in T_{x} \mathcal{D}, X(x) \in T_{x} \mathcal{D}$ and $X^{V}\left(v_{x}\right) \in T_{v_{x}} T \mathcal{D}$ is its vertical lift to $v_{x}$. Locally,

$$
\Psi\left(q^{i}, y^{A}, \dot{q}^{i}, \dot{y}^{A}, \mu_{i}, \mu_{A}, \gamma_{i}, \gamma_{A}\right)=\left(q^{i}, y^{A}, \gamma_{i}, \gamma_{A}\right)
$$

Definition 8 Define the Legendre transform associated with a second-order nonholonomic system $\left(\mathcal{D}^{(2)}, \Sigma_{\mathcal{L}}\right)$ as the map $\mathbb{F} \mathcal{L}: \Sigma_{\mathcal{L}} \rightarrow T^{*} \mathcal{D}$ given by $\mathbb{F} \mathcal{L}=\Psi \circ i_{\Sigma_{\mathcal{L}}}$. In local coordinates, it is given by

$$
\mathbb{F} \mathcal{L}\left(q^{i}, y^{A}, \dot{y}^{A}, \gamma_{i}\right)=\left(q^{i}, y^{A}, \gamma_{i}, \frac{\partial \mathcal{L}}{\partial \dot{y}^{A}}\right)
$$


The following diagram summarizes the situation

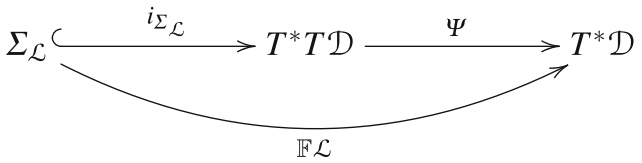

Definition 9 We say that the second-order nonholonomic system $\left(\mathcal{D}^{(2)}, \Sigma_{\mathcal{L}}\right)$ is regular if $\mathbb{F} \mathcal{L}: \Sigma_{\mathcal{L}} \rightarrow T^{*} \mathcal{D}$ is a local diffeomorphism and hyperregular if $\mathbb{F} \mathcal{L}$ is a global diffeomorphism.

From the local expression of $\mathbb{F} \mathcal{L}$ we can observe that from a direct application of the implicit function theorem we have

Proposition 2 The second-order nonholonomic system $\left(\mathcal{D}^{(2)}, \Sigma_{\mathcal{L}}\right)$ determined by $\mathcal{L}: \mathcal{D}^{(2)} \rightarrow \mathbb{R}$ is regular if and only if the matrix $\left(\frac{\partial^{2} \mathcal{L}}{\partial \dot{y}^{A} \partial \dot{y}^{B}}\right)$ is non singular.

Remark 7 Observe that if the Lagrangian $\mathcal{L}: \mathcal{D}^{(2)} \rightarrow \mathbb{R}$ is determined from an optimal control problem and its expression is given by (3), then the regularity of the matrix $\left(\frac{\partial^{2} \mathcal{L}}{\partial \dot{y}^{A} \partial \dot{y}^{B}}\right)$ is equivalent to $\operatorname{det}\left(\frac{\partial^{2} C}{\partial u^{A} \partial u^{B}}\right) \neq 0$ for the cost function.

\subsection{Hamiltonian Formalism}

Assume that the system is regular. Let $p_{i}=\gamma_{i}$ and $p_{A}=\frac{\partial \mathcal{L}}{\partial \dot{y}^{A}}$, then we can write $\dot{y}^{A}=\dot{y}^{A}\left(q^{i}, y^{A}, p_{A}\right)$. Define the Hamiltonian function $\mathcal{H}: T^{*} \mathcal{D} \rightarrow \mathbb{R}$ by

$$
\mathcal{H}(\alpha)=\left\langle\alpha,\left.\pi_{T^{*} T \mathcal{D}}\right|_{\Sigma_{\mathcal{L}}}\left(\mathbb{F} \mathcal{L}^{-1}(\alpha)\right)\right\rangle-\mathcal{L}\left(\left.\pi_{T^{*} T \mathcal{D}}\right|_{\Sigma_{\mathcal{L}}}\left(\mathbb{F} \mathcal{L}^{-1}(\alpha)\right)\right),
$$

where $\alpha \in T^{*} \mathcal{D}$ is a 1 -form on $\mathcal{D}$, and $\left.\pi_{T^{*} T \mathcal{D}}\right|_{\Sigma_{\mathcal{L}}}: \Sigma_{\mathcal{L}} \rightarrow \mathcal{D}^{(2)}$ is the projection locally given by $\left.\pi_{T^{*} T \mathcal{D}}\right|_{\Sigma_{\mathcal{L}}}\left(q^{i}, y^{A}, \dot{y}^{A}, \gamma_{i}\right)=\left(q^{i}, y^{A}, \dot{y}^{A}\right)$. Locally, the Hamiltonian is given by

$$
\left.\mathcal{H}\left(q^{i}, y^{A}, p_{i}, p_{A}\right)=p_{A} \dot{y}^{A}\left(q^{i}, y^{A}, p_{A}\right)\right)+p_{i} \rho_{A}^{i} y^{A}-\mathcal{L}\left(q^{i}, y^{A}, \dot{y}^{A}\left(q^{i}, y^{A}, p_{A}\right)\right),
$$

where we are using

$$
\mathbb{F} \mathcal{L}^{-1}\left(q^{i}, y^{A}, p_{i}, p_{A}\right)=\left(q^{i}, y^{A}, \rho_{A}^{i}, \dot{y}^{A}\left(q^{i}, y^{A}, p_{A}\right), \frac{\partial \mathcal{L}}{\partial q^{i}}-p_{j} \frac{\partial \rho_{A}^{j}}{\partial q^{i}} y^{A}, \frac{\partial \mathcal{L}}{\partial y^{A}}-p_{j} \rho_{A}^{j}, p_{i}, p_{A}\right)
$$


Below, we will see that the dynamics of the nonholonomic optimal control problem is determined by the Hamiltonian system given by the triple $\left(T^{*} \mathcal{D}, \omega_{\mathcal{D}}, \mathcal{H}\right)$, where $\omega_{\mathcal{D}}$ is the standard symplectic 2 -form on $T^{*} \mathcal{D}$.

The dynamics of the optimal control problem for the second-order nonholonomic system are given by the symplectic hamiltonian dynamics determined by the equation

$$
i_{X_{\mathcal{H}}} \omega_{\mathcal{D}}=d \mathcal{H} .
$$

Therefore, if we consider the integral curves of $X_{\mathcal{H}}$, they are of the type $t \mapsto$ $\left(\dot{q}^{i}(t), \dot{y}^{A}(t), \dot{p}_{i}(t), \dot{p}_{A}(t)\right)$; the solutions of the nonholonomic Hamiltonian system are specified by the Hamilton's equations on $T^{*} \mathcal{D}$

$$
\begin{aligned}
\dot{q}^{i} & =\frac{\partial \mathcal{H}}{\partial p_{i}}, & \dot{y}^{A} & =\frac{\partial \mathcal{H}}{\partial p_{A}}, \\
\dot{p}_{i} & =-\frac{\partial \mathcal{H}}{\partial q^{i}}, & \dot{p}_{A} & =-\frac{\partial \mathcal{H}}{\partial y^{A}},
\end{aligned}
$$

i.e.,

$$
\begin{aligned}
\dot{q}^{i} & =\rho_{A}^{i} y^{A}, \\
\dot{p}_{i} & =\frac{\partial \mathcal{L}}{\partial q^{i}}\left(q^{i}, y^{A}, \dot{y}^{A}\left(q^{i}, y^{A}, p_{A}\right)\right)-p_{j} \frac{\partial \rho_{A}^{j}}{\partial q^{i}} y^{A}, \\
\dot{p}_{A} & =\frac{\partial \mathcal{L}}{\partial y^{A}}\left(q^{i}, y^{A}, \dot{y}^{A}\left(q^{i}, y^{A}, p_{A}\right)\right)-p_{j} \rho_{A}^{j} .
\end{aligned}
$$

From (7) it is clear that the flow preserves the symplectic 2-form $\omega_{\mathcal{D}}$. Moreover, these equations are equivalent to the equations given in (4) using the identification of the Lagrange multipliers with the variables $p_{i}$ and the relation $p_{A}=\frac{\partial \mathcal{L}}{\partial \dot{y}^{A}}$.

Remark 8 We observe that in our formalism the optimal control dynamics are deduced using a constrained variational procedure and equivalently it is possible to apply the Hamilton-Pontryagin's principle (see e.g., [19]), but, in any case, this "variational procedure" implies the preservation of the symplectic 2-form, and this is reflected in the Lagrangian submanifold character. Moreover, in our case, under the regularity condition, we have seen that the Lagrangian submanifold approach shows that the system can be written as a Hamiltonian system (which is obviously symplectic).

Additionally, we use the Lagrangian submanifold $\Sigma_{\mathcal{L}}$ as a way to define intrinsically the Hamiltonian side since we define the Legendre transformation using the Lagrangian submanifold $\Sigma_{\mathcal{L}}$. However there are other possibilities. For instance, 
in [1] (Sect. 4.2) the authors define the corresponding momenta for a vakonomic system. Using this procedure the momenta are locally expressed as follows

$$
\begin{aligned}
p_{i} & =\frac{\partial \tilde{\mathcal{L}}}{\partial \dot{q}^{i}}+\lambda^{j} \frac{\partial f_{j}}{\partial \dot{q}^{i}}, \\
p_{A} & =\frac{\partial \tilde{\mathcal{L}}}{\partial \dot{y}^{A}}+\lambda^{j} \frac{\partial f^{j}}{\partial \dot{y}^{A}},
\end{aligned}
$$

where $\tilde{\mathcal{L}}$ is an arbitrary extension of $\mathcal{L}$ to $T \mathcal{D}$ and $f^{j}=\dot{q}^{j}-\rho_{A}^{j} y^{A}=0$ are the constraint equations. A simple computation shows that both approaches are equivalent, but our derivation is more intrinsic and geometric, i.e., independent of coordinates or extensions and without using Lagrange multipliers.

\subsection{Example: Continuously Variable Transmission (CVT) (cont'd)}

Here we continue the example of the optimal control problem of a continuously variable transmission that we considered in Sect.3.1 Recall that the constraint distribution for the CVT is given by $\mathcal{D} \subset T\left(\mathbb{S}^{1} \times \mathbb{S}^{1} \times \mathbb{R}\right)$, where

$$
\mathcal{D}=\operatorname{span}\left\{\frac{1}{m} \frac{\partial}{\partial x},(1-x) \frac{\partial}{\partial \theta_{1}}+x \frac{\partial}{\partial \theta_{2}}\right\} .
$$

The system is regular since

$$
\operatorname{det}\left(\frac{\partial^{2} \mathcal{L}}{\partial \dot{y}^{A} \partial \dot{y}^{B}}\right)=\frac{(B(x))^{2}}{m^{2}} \neq 0,
$$

because $B(x)=J_{1}(1-x)^{2}+J_{2} x^{2} \neq 0$.

Denoting by $\left(\theta_{1}, \theta_{2}, x, y_{1}, y_{2}, p_{\theta_{1}}, p_{\theta_{2}}, p_{x}, p_{1}, p_{2}\right)$, the local coordinates on $T^{*} \mathcal{D}$, the dynamics of the optimal control problem for this nonholonomic system are determined by the Hamiltonian $\mathcal{H}: T^{*} \mathcal{D} \rightarrow \mathbb{R}$, given by

$$
\begin{aligned}
\mathcal{H}\left(\theta, \theta_{2}, x, y_{1}, y_{2}, p_{\theta_{1}}, p_{\theta_{2}}, p_{x}, p_{1}, p_{2}\right) & =\frac{m^{2} p_{1}^{2}}{2}+\frac{p_{2}^{2}}{2(B(x))^{2}}+\frac{p_{2} A(x) y_{1} y_{2}}{m B(x)} \\
& +p_{\theta_{1}}(1-x) y_{2}+p_{\theta_{2}} x y_{2}+p_{x} \frac{y_{1}}{m}
\end{aligned}
$$

The corresponding Hamiltonian equations of motion are

$$
\begin{aligned}
& \dot{y}_{1}=m^{2} p_{1}, \quad \dot{p}_{\theta_{1}}=0, \\
& \dot{y}_{2}=\frac{p_{2}}{(B(x))^{2}}+\frac{A(x) y_{1} y_{2}}{m B(x)}, \quad \dot{p}_{\theta_{2}}=0,
\end{aligned}
$$




$$
\begin{aligned}
& \dot{p}_{x}=y_{2}\left(p_{\theta_{1}}-p_{\theta_{2}}\right)-\frac{p_{2} y_{1} y_{2}\left((A(x))^{2}-J_{1} J_{2}\right)}{m(B(x))^{2}}-\frac{2 p_{2}^{2} A(x)}{(B(x))^{3}}, \\
& \dot{p}_{1}==-\frac{p_{2} A(x) y_{2}}{m B(x)}-\frac{p_{x}}{m}, \quad \dot{p}_{2}=-\frac{p_{2} A(x) y_{1}}{m B(x)}-p_{\theta_{1}}(1-x)-p_{\theta_{2}} x .
\end{aligned}
$$

\subsection{Example: Chaplygin Sleigh (cont'd)}

In what follows, we continue the example of the optimal control problem of the Chaplygin sleigh that we studied in Sect. 3.2. Recall that the constraint distribution is given by $\mathcal{D} \subset \operatorname{TSE}(2)$, where

$$
\mathcal{D}=\operatorname{span}\left\{\frac{1}{J} \frac{\partial}{\partial \theta}, \frac{\cos \theta}{m} \frac{\partial}{\partial x}+\frac{\sin \theta}{m} \frac{\partial}{\partial y}\right\} .
$$

The system is regular since

$$
\operatorname{det}\left(\frac{\partial^{2} \mathcal{L}}{\partial \dot{y}^{A} \partial \dot{y}^{B}}\right)=\frac{a^{4}}{J^{4}} \neq 0 .
$$

Denoting by $\left(x, y, \theta, y_{1}, y_{2}, p_{x}, p_{y}, p_{\theta}, p_{1}, p_{2}\right)$, the local coordinates on $T^{*} \mathcal{D}$, the dynamics of the optimal control problem for this nonholonomic system are determined by the Hamiltonian $\mathcal{H}: T^{*} \mathcal{D} \rightarrow \mathbb{R}$, given by $\mathcal{H}\left(x, y, \theta, y_{1}, y_{2}, p_{x}, p_{y}, p_{\theta}, p_{1}, p_{2}\right)=\frac{J^{2}}{2 b^{2}} p_{1}^{2}+\frac{m^{2}}{2} p_{2}^{2}+p_{x} \frac{\cos \theta}{m} y_{2}+\frac{p_{\theta}}{J} y_{1}+p_{y} \frac{\sin \theta}{m} y_{2}$.

The Hamiltonian equations of motion are

$$
\begin{aligned}
& \dot{y}_{1}=\frac{J^{2} p_{1}}{b^{2}}, \quad \dot{y}_{2}=m^{2} p_{2}, \quad \dot{p}_{x}=0, \quad \dot{p}_{y}=0, \\
& \dot{p}_{\theta}=p_{x} \frac{\sin \theta}{m} y_{2}-p_{y} \frac{\cos \theta}{m} y_{2}, \\
& \dot{p}_{1}=-\frac{p_{\theta}}{J}, \quad \dot{p}_{2}=-p_{x} \frac{\cos \theta}{m}-p_{y} \frac{\sin \theta}{m} .
\end{aligned}
$$

Integrating the equations $\dot{p}_{x}=0$ and $\dot{p}_{y}=0$ give $p_{x}=c_{1}$ and $p_{y}=c_{2}$, where $c_{1}$ and $c_{2}$ are constants, the above equations reduce to the following equations

$$
\begin{aligned}
& \dot{y}_{1}=\frac{J^{2} p_{1}}{b^{2}}, \quad \dot{p}_{\theta}=c_{1} \frac{\sin \theta}{m} y_{2}-c_{2} \frac{\cos \theta}{m} y_{2}, \\
& \dot{y}_{2}=m^{2} p_{2}, \quad \dot{p}_{1}=-\frac{p_{\theta}}{J}, \quad \dot{p}_{2}=-c_{1} \frac{\cos \theta}{m}-c_{2} \frac{\sin \theta}{m} .
\end{aligned}
$$


Differentiating the equations for $\dot{y}_{1}$ and $\dot{y}_{2}$ and substituting in the above equations, we obtain

$$
\frac{\dddot{y}_{1}}{J}=\frac{y_{2}}{m b^{2}}\left(c_{2} \cos \theta-c_{1} \sin \theta\right), \quad \ddot{y}_{2}=-m\left(c_{1} \cos \theta+c_{2} \sin \theta\right),
$$

which are the same as the ones obtained in the Lagrangian setting.

Observe that in the case of motion planning for obstacle avoidance, the Hamiltonian $\mathcal{H}: T^{*} \mathcal{D} \rightarrow \mathbb{R}$, is given by

$$
\begin{aligned}
\mathcal{H}\left(x, y, \theta, y_{1}, y_{2}, p_{x}, p_{y}, p_{\theta}, p_{1}, p_{2}\right) & =\frac{J^{2}}{2 b^{2}} p_{1}^{2}+\frac{m^{2}}{2} p_{2}^{2}+p_{x} \frac{\cos \theta}{m} y_{2}+\frac{p_{\theta}}{J} y_{1}+p_{y} \frac{\sin \theta}{m} y_{2} \\
& -\frac{\kappa}{2\left(x-x_{C}\right)^{2}+2\left(y-y_{C}\right)^{2}} .
\end{aligned}
$$

The corresponding Hamiltonian equations of motion are

$$
\begin{aligned}
& \dot{y}_{1}=\frac{J^{2} p_{1}}{b^{2}}, \quad \dot{y}_{2}=m^{2} p_{2}, \quad \dot{p}_{x}=\frac{\kappa\left(x-x_{C}\right)}{\left(\left(x-x_{C}\right)^{2}+\left(y-y_{C}\right)^{2}\right)^{2}}, \\
& \dot{p}_{y}=\frac{\kappa\left(y-y_{C}\right)}{\left(\left(x-x_{C}\right)^{2}+\left(y-y_{C}\right)^{2}\right)^{2}}, \quad \dot{p}_{\theta}=p_{x} \frac{\sin \theta}{m} y_{2}-p_{y} \frac{\cos \theta}{m} y_{2}, \\
& \dot{p}_{1}==-\frac{p_{\theta}}{J}, \quad \dot{p}_{2}=-p_{x} \frac{\cos \theta}{m}-p_{y} \frac{\sin \theta}{m} .
\end{aligned}
$$

\section{Conclusions and Future Research}

In this section we summarize the contributions of our work and discuss possible future research.

\subsection{Conclusions}

In this paper, we studied optimal control problems for a class of nonholonomic mechanical systems. We have given a geometrical derivation of the equations of motion of a nonholonomic optimal control problem as a constrained variational problem on the tangent space of the constraint distribution. We have seen how the dynamics of the optimal control problem can be completely described by a Lagrangian submanifold of an appropriate cotangent bundle and under some mild regularity conditions, we have derived the equations of motion for the nonholonomic optimal control problem as a classical set of Hamilton's equations on the cotangent bundle of the constraint distribution. We have introduced the notion of Legendre 
transformation in this context to establish the relationship between the Lagrangian and Hamiltonian dynamics. We applied our techniques to different examples: optimal control of the Chaplygin sleigh, a continuously variable transmission and a problem of motion planning for obstacle avoidance.

\subsection{Future Research: Construction of Geometric and Variational Integrators for Optimal Control Problems of Nonholonomic Mechanical Systems}

We have seen that an optimal control problem of a nonholonomic system may be viewed as a Hamiltonian system on $T^{*} \mathcal{D}$. One can thus use standard methods for symplectic integration such as symplectic Runge-Kutta methods, collocation methods, Störmer-Verlet, symplectic Euler methods, etc., developed and studied in [26-28, 40], e.g., to simulate nonholonomic optimal control problems.

We would like to use the theory of variational integrators as an alternative way to construct integration schemes for these kinds of optimal control problems following the results given in Sect. 3. Recall that in the continuous case we have considered a Lagrangian function $\mathcal{L}: \mathcal{D}^{(2)} \rightarrow \mathbb{R}$. Since $\mathcal{D}^{(2)}$ is a subset of $T \mathcal{D}$ we can discretize the tangent bundle $T \mathcal{D}$ by the cartesian product $\mathcal{D} \times \mathcal{D}$. Therefore, our discrete variational approach for optimal control problems of nonholonomic mechanical systems will be determined by the construction of a discrete Lagrangian $\mathcal{L}_{d}: \mathcal{D}_{d}^{(2)} \rightarrow \mathbb{R}$ where $\mathcal{D}_{d}^{(2)}$ is the subset of $\mathcal{D} \times \mathcal{D}$, locally determined by imposing the discretization of the constraint $\dot{q}^{i}=\rho_{A}^{i}(q) y^{A}$, for instance we can consider

$$
\mathcal{D}_{d}^{(2)}=\left\{\left(q_{0}^{i}, y_{0}^{A}, q_{1}^{i}, y_{1}^{A}\right) \in \mathcal{D} \times \mathcal{D} \mid \frac{q_{1}^{i}-q_{0}^{i}}{h}=\rho_{A}^{i}\left(\frac{q_{0}^{i}+q_{1}^{i}}{2}\right)\left(\frac{y_{0}^{A}+y_{1}^{A}}{2}\right)\right\} .
$$

Now, the system is in a form appropriate for the application of discrete variational methods for constrained systems (see [34] and references therein).

Acknowledgements This work has been partially supported by grants MTM 2013-42870-P, MTM 2009-08166-E, IRSES-project "Geomech-246981" and NSF grants INSPIRE-1363720 and DMS1207693. We wish to thank Klas Modin and Olivier Verdier for allowing us to use their description of the Continuously Variable Transmission Gearbox.

\section{References}

1. Arnold, V.: Dynamical Systems, vol. III. Springer, New York/Heidelberg/Berlin (1988)

2. Balseiro, P., de León, M., Marrero, J.C., Martín de Diego, D.: The ubiquity of the symplectic Hamiltonian equations in mechanics. J. Geom. Mech. 1, 11-34 (2009)

3. Barbero Liñan, M., de León, M., Marrero, J.C., Martín de Diego, D., Muñoz Lecanda, M.: Kinematic reduction and the Hamilton-Jacobi equation. J. Geom. Mech. 3, 207-237 (2012) 
4. Bloch, A.M.: Nonholonomic Mechanics and Control. Interdisciplinary Applied Mathematics Series, vol. 24. Springer, New York (2003)

5. Bloch, A., Crouch, P.: Nonholonomic and vakonomic control systems on Riemannian manifolds. In: Enos, M.J. (ed.) Dynamics and Control of Mechanical Systems. Fields Institute Communications, vol. 1, pp. 25-52. AMS, Providence (1993)

6. Bloch, A., Crouch, P.: Nonholonomic control systems on Riemannian manifolds. SIAM J. Control Optim. 33, 126-148 (1995)

7. Bloch, A., Crouch, P.: On the equivalence of higher order variational problems and optimal control problems. In: Proceedings of the IEEE International Conference on Decision and Control, Kobe, pp. 1648-1653 (1996)

8. Bloch, A., Crouch, P.: Optimal control, optimization, and analytical mechanics. In: Baillieul, J., Willems, J.C. (eds.) Mathematical Control Theory, pp. 268-321. Springer, New York (1998)

9. Bloch, A., Hussein, I.: Optimal control of underactuated nonholonomic mechanical systems. IEEE Trans. Autom. Control 53, 668-682 (2008)

10. Bloch, A., Marsden, J., Zenkov D.: Nonholonomic mechanics. Not. AMS 52, 324-333 (2005)

11. Bloch, A., Marsden, J., Zenkov, D.: Quasivelocities and symmetries in non-holonomic systems. Dyn. Syst. 24(2), 187-222 (2009)

12. Borisov, A., Mamaev, I.: On the history of the development of the nonholonomic dynamics. Regul. Chaot. Dyn. 7(1), 43-47 (2002)

13. Bullo, F., Lewis, A.: Geometric Control of Mechanical Systems: Modeling, Analysis, and Design for Simple Mechanical Control Systems. Texts in Applied Mathematics. Springer, New York (2005)

14. Chaplygin, S.: On the theory of motion of nonholonomic systems. The theorem on the reducing multiplier. Math. Sbornik XXVIII, 303-314 (1911, in Russian)

15. Cortés, J., Martínez, E.: Mechanical control systems on Lie algebroids. IMA J. Math. Control. Inform. 21, 457-492 (2004)

16. Cortés, J., de León, M., Marrero, J.C., Martínez, E.: Nonholonomic Lagrangian systems on Lie algebroids. Discret. Cont. Dyn. Syst. Ser. A 24(2), 213-271 (2009)

17. Grabowska, K., Grabowski, J., Urbanski, P.: Geometrical mechanics on algebroids. Int. J. Geom. Methods Mod. Phys. 3(3), 559-575 (2006)

18. Grabowski, J., de León, M., Marrero, J.C., Martín de Diego, D.: Nonholonomic constraints: a new viewpoint. J. Math. Phys. 50(1), 013520, 17 pp. (2009)

19. Holm, D.: Geometric mechanics. Part I and II. Imperial College Press, London; distributed by World Scientific Publishing Co. Pte. Ltd., Hackensack (2008)

20. Jurdjevic, V.: Geometric Control Theory. Cambridge Studies in Advanced Mathematics, vol. 52. Cambridge University Press (1997)

21. Jurdjevic, V.: Optimal control, geometry and mechanics. In: Baillieul, J., Willems, J.C. (eds.) Mathematical Control Theory, pp. 227-267. Springer, New York (1998)

22. Kelly, S., Murray, R.: Geometric phases and robotic locomotion. J. Robot. Syst. 12, 417-431 (1995)

23. Khatib, O.: Real-time robots obstacle avoidance for manipulators and mobile robots. Int. J. Robot. 5(1), 90-98 (1986)

24. Kodistschek, D.E., Rimon, E.: Robot navigation function on manifolds with boundary. Adv. Appl. Math. 11(4), 412-442 (1980)

25. Koiller, J.: Reduction of some classical non-holonomic systems with symmetry. Arch. Ration. Mech. Anal. 118, 113-148 (1992)

26. Leimkuhler, B., Reich, S.: Symplectic integration of constrained Hamiltonian systems. Math. Comput. 63, 589-605 (1994)

27. Leimkuhler, B., Reich, S.: Simulating Hamiltonian Dynamics. Cambridge University Press, Cambridge (2004)

28. Leimkmuhler, B., Skeel, R.: Symplectic numerical integrators in constrained Hamiltonian systems. J. Comput. Phys. 112, 117-125 (1994)

29. de León, M.: A historical review on nonholonomic mechanics. RACSAM 106, 191-224 (2012) 
30. de León, M., Martín de Diego, D.: On the geometry of non-holonomic Lagrangian systems. J. Math. Phys. 37(7), 3389-3414 (1996)

31. de León, M., Rodrigues, P.R.: Generalized Classical Mechanics and Field Theory. North-Holland Mathematical Studies, vol. 112. North-Holland, Amsterdam (1985)

32. Lewis, A.D.: Simple mechanical control systems with constraints. IEEE Trans. Autom. Control 45, 1420-1436 (2000)

33. Libermann, P., Marle, Ch-M.: Symplectic Geometry and Analytical Mechanics. Mathematics and its Applications, vol. 35. D. Reidel Publishing Co., Dordrecht (1987)

34. Marrero, J.C., Martín de Diego, D., Stern, A.: Symplectic groupoids and discrete constrained Lagrangian mechanics. Discret. Cont. Dyn. Syst. Ser. A 35(1), 367-397 (2015)

35. Maruskin, J.M., Bloch, A., Marsden, J.E., Zenkov, D.V.: A fiber bundle approach to the transpositional relations in nonholonomic mechanics. J. Nonlinear Sci. 22(4), 431-461 (2012)

36. Modin, K., Verdier, O.: Integrability of nonholonomically coupled oscillators. J. Discret. Cont. Dyn. Syst. 34(3), 1121-1130 (2014)

37. Murray, R., Sastry, S.S.: Nonholonomic motion planning: steering using sinusoids. IEEE Trans. Autom. Control 38, 700-716 (1993)

38. Neimark, J.I., Fufaev, N.A.: Dynamics of Nonholonomic Systems. Translations of Mathematical Monographs, vol. 33. American Mathematical Society, Providence (1972)

39. Ostrowski, J.P.: Computing reduced equations for robotic systems with constraints and symmetries. IEEE Trans. Robot. Autom. 15(1), 111-123 (1999)

40. Sanz-Serna, J.M., Calvo, M.P.: Numerical Hamiltonian Problems. Applied Mathematics and Mathematical Computation, vol. 7. Chapman and Hall, London (1994)

41. Sniatycki, J., Tulczyjew, W.M.: Generating forms of Lagrangian submanifolds. Indiana Univ. Math. J. 22(3), 267-275 (1972)

42. Sussman, H.: Geometry and optimal control. In: Baillieul, J., Willems, J.C. (eds.) Mathematical Control Theory, pp. 140-198. Springer, New York (1998)

43. Sussman, H., Jurdjevic, V.: Contollability of nonlinear systems. J. Differ. Equ. 12, 95116 (1972)

44. Tulczyjew, W.M.: Les sous-variétés lagrangiennes et la dynamique hamiltonienne. C. R. Acad. Sci. Paris Série A 283, 15-18 (1976)

45. Tulczyjew, W.M.: Les sous-variétés lagrangiennes et la dynamique lagrangienne. C. R. Acad. Sci. Paris Série A 283, 675-678 (1976)

46. Weinstein, A.: Lectures on Symplectic Manifolds. CBMS Regional Conference Series in Mathematics, vol. 29. American Mathematical Society, Providence (1979) 